\title{
Homological dimensions of smooth crossed products
}

\author{
Petr Kosenko
}

May 22, 2019

\begin{abstract}
In this paper we provide upper estimates for the global projective dimensions of smooth crossed products $\mathscr{S}(G, A ; \alpha)$ for $G=\mathbb{R}$ and $G=\mathbb{T}$ and a self-induced Fréchet-Arens-Michael algebra $A$. In order to do this, we provide a powerful generalization of methods which are used in the works of Ogneva and Helemskii.
\end{abstract}

\section{Introduction}

There are numerous papers dedicated to homological properties of smooth crossed products of Fréchet algebras and $\mathrm{C}^{*}$-algebras, see [Sch93], PS94], [Mey04], GG11], or [Nes14], for example.

However, it seems that nothing is known about homological dimensions of smooth crossed products. In the paper [Kos17] we provided the estimates for homological dimensions of holomorphic Ore extensions and smooth crossed products by $\mathbb{Z}$ of unital $\hat{\otimes}$-algebras, and in this paper we show that the methods of the author's previous works and the paper [OK84] can be adapted to smooth crossed products by $\mathbb{R}$ and $\mathbb{T}$.

The idea behind the estimates lies in the construction of admissible $\widehat{\Omega}^{1}$-like sequences for the required non-unital algebras. What do we mean by that? Recall the definition of a bimodule of relative 1-forms:

Definition 0.1. Let $A$ be an algebra and $X$ be an $A$-bimodule. A linear map $d: A \longrightarrow X$ is called an A-derivation if

$$
d(a b)=d(a) \circ b+a \circ d(b)
$$

for every $a, b \in A$.

Definition 0.2. Let $R$ be a unital $\hat{\otimes}$-algebra, and let $A$ denote a unital $R$ - $\hat{\otimes}$-algebra (see Definition 3.1). A pair $\left(\widehat{\Omega}_{R}^{1}(A), d_{A}\right)$, which consists of a $A$ - $\hat{\otimes}$-bimodule $\widehat{\Omega}_{R}^{1}(A)$ and a continuous $R$-derivation $d_{A}: A \longrightarrow \widehat{\Omega}_{R}^{1}(A)$, is called the bimodule of relative 1-forms of $A$, if this pair is universal in the following sense:

for every $A$ - $\hat{\otimes}$-bimodule $M$ and a continuous $R$-derivation $D: A \longrightarrow M$ there exists a unique continuous $A$ - $\hat{\otimes}$-bimodule homomorphism $\widetilde{D}: \widehat{\Omega}_{R}^{1}(A) \longrightarrow M$ such that $D=\widetilde{D} \circ d_{A}$.

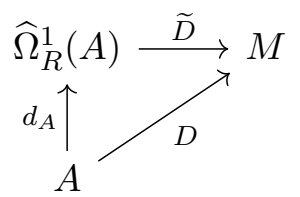

This construction is a topological version of a construction presented in [CQ95]. It is not hard to prove that $\widehat{\Omega}_{R}^{1}(A)$ is a well-defined object, moreover, this bimodule is a part of an extremely useful admissible sequence. The following theorem is the topological version of [CQ95, Proposition 2.5].

Theorem 0.1 ([Pir08], Proposition 7.2). Let $R$ be a unital $\hat{\otimes}$-algebra and let $A$ denote a unital $R$ $\hat{\otimes}$-algebra. Then there exists a sequence which splits in the categories $A-R$ - $\hat{\otimes}$-mod and $R$ - $A$ - $\hat{\otimes}$-mod:

$$
0 \longrightarrow \widehat{\Omega}_{R}^{1}(A) \stackrel{j}{\longrightarrow} A \hat{\otimes}_{R} A \stackrel{m}{\longrightarrow} A \longrightarrow 0,
$$

where $m(a \otimes b)=a b$. In particular, this sequence is admissible. 
In the paper [Kos17] we utilized the sequence 1 in order to obtain the upper estimates for the homological dimensions of different types of non-commutative Ore-like extensions. This sequence proves to be quite useful because in the unital case for the extensions we study it turns out that $\widehat{\Omega}_{R}^{1}(A) \cong A \hat{\otimes}_{R} A$ as a $R$ - $\hat{\otimes}$-module.

However, when $G=\mathbb{R}$ or $G=\mathbb{T}$, then for a Fréchet-Arens-Michael algebra $A$ the algebras $\mathscr{S}(G, A ; \alpha)$ are, in general, not unital. Nevertheless, we managed to obtain the exact sequences for these algebras, which look similar to the (1), and which allowed us to derive the upper estimates for the global projective dimensions of $\mathscr{S}(\mathbb{R}, A ; \alpha)$ and $\mathscr{S}(\mathbb{T}, A ; \alpha)$.

We conjecture that estimates for homological dimensions should look as follows:

Conjecture 0.1. Let $A$ be a Fréchet-Arens-Michael algebra (not necessarily unital) with a smooth $m$-tempered action $\alpha$ of $\mathbb{R}$ or $\mathbb{T}$ on $A$. Denote the left (projective) global dimension by dgl. Then for $G=\mathbb{R}$ or $G=\mathbb{T}$ we have

$$
\operatorname{dgl}(A) \leq \operatorname{dgl}(\mathscr{S}(G, A ; \alpha)) \leq \operatorname{dgl}(A)+1
$$

The main results of this paper are Theorems 2.2, 2.3, 3.2, 3.3, In particular, we have proven the weak form of the above conjecture.

Theorem 0.2. Let $A$ be a Fréchet-Arens-Michael algebra, which satisfies the following condition: the multiplication map $m: A \hat{\otimes}_{A} A \longrightarrow A$ is a $A$ - $\hat{\otimes}$-bimodule isomorphism. Also let $\alpha$ denote a smooth $m$-tempered action of $\mathbb{R}$ or $\mathbb{T}$ on $A$. Denote the left (projective) global dimension by dgl. Then for $G=\mathbb{R}$ or $G=\mathbb{T}$ we have

$$
\operatorname{dgl}(\mathscr{S}(G, A ; \alpha)) \leq \max \{\operatorname{dgl}(A), 1\}+1
$$

\section{Preliminaries}

\section{$1.1 \quad$ Notation}

Remark. All algebras in this paper are defined over the field of complex numbers and assumed to be associative. Unlike in the paper [Kos17], here we allow the algebras are to be non-unital.

Definition 1.1. A Fréchet space is a complete metrizable locally convex space.

Let us introduce some notation (see [Hel86] and [Pir12] for more details). Denote by LCS, Fr the categories of complete locally convex spaces, Fréchet spaces, respectively. Also we will denote the category of vector spaces by Lin.

For a locally convex Hausdorff space $E$ we will denote its completion by $\tilde{E}$. Also for locally convex Hausdorff spaces $E, F$ the notation $E \hat{\otimes} F$ denotes the completed projective tensor product of $E, F$.

By $A_{+}$we will denote the unitization of an algebra $A$. By $A^{\text {op }}$ we denote the opposite algebra.

Definition 1.2. A complete locally convex algebra with jointly continuous multiplication is called a $\hat{\otimes}$-algebra.

A $\hat{\otimes}$-algebra with the underlying locally compact space which is a Fréchet space is called a Fréchet algebra.

Definition 1.3. A locally convex algebra $A$ is called $m$-convex if the topology on it can be defined by a family of submultiplicative seminorms.

Definition 1.4. A complete locally $m$-convex algebra is called an Arens-Michael algebra.

Definition 1.5. Let $A$ be a $\hat{\otimes}$-algebra and let $M$ be a complete locally convex space which is also a left $A$-module. Also suppose that the natural map $A \times M \rightarrow M$ is jointly continuous. Then we will call $M$ a left $A$ - $\hat{\otimes}$-module. In a similar fashion we define right $A$ - $\hat{\otimes}$-modules and $A$ - $B$ - $\hat{\otimes}$-bimodules.

A $\hat{\otimes}$-module over a Fréchet algebra which is itself a Fréchet space is called a Fréchet $A$ - $\hat{\otimes}$-module. 
For arbitrary $\hat{\otimes}$-algebras $A, B$ we denote

$$
\begin{aligned}
A-\bmod & =\text { the category of left } A-\hat{\otimes} \text {-modules } \\
\bmod -A & =\text { the category of right } A-\hat{\otimes} \text {-modules, } \\
A-\bmod -B & =\text { the category of } A-B-\hat{\otimes} \text {-bimodules. }
\end{aligned}
$$

For unital $\hat{\otimes}$-algebras $A, B$ we denote

$$
\begin{aligned}
A \text {-unmod } & =\text { the category of unital left } A \text { - } \hat{\otimes} \text {-modules } \\
\text { unmod- } A & =\text { the category of unital right } A \text { - } \hat{\otimes} \text {-modules, } \\
A \text {-unmod- } B & =\text { the category of unital } A-B \text { - } \hat{\otimes} \text {-bimodules. }
\end{aligned}
$$

Let $A$ be a $\hat{\otimes}$-algebra, and consider a complex of $A$ - $\hat{\otimes}$-modules:

$$
\ldots \stackrel{d_{n+1}}{\longrightarrow} M_{n+1} \stackrel{d_{n}}{\longrightarrow} M_{n} \stackrel{d_{n-1}}{\longrightarrow} M_{n-1} \stackrel{d_{n-2}}{\longrightarrow} \ldots
$$

then we will denote this complex by $\{M, d\}$.

Definition 1.6. Let $A$ be a $\hat{\otimes}$-algebra and consider a left $A$ - $\hat{\otimes}$-module $Y$ and a right $A$ - $\hat{\otimes}$-module $X$.

(1) A bilinear map $f: X \times Y \longrightarrow Z$, where $Z \in \mathbf{L C S}$, is called $A$-balanced if $f(x \circ a, y)=f(x, a \circ y)$ for every $x \in X, y \in Y, a \in A$.

(2) A pair $\left(X \hat{\otimes}_{A} Y, i\right)$, where $X \hat{\otimes}_{A} Y \in \mathbf{L C S}$, and $i: X \times Y \longrightarrow X \hat{\otimes}_{A} Y$ is a continuous $A$-balanced map, is called the completed projective tensor product of $X$ and $Y$, if for every $Z \in \mathbf{L C S}$ and continuous $A$-balanced map $f: X \times Y \longrightarrow Z$ there exists a unique continuous linear map $\tilde{f}: X \hat{\otimes}_{A} Y \longrightarrow Z$ such that $f=\tilde{f} \circ i$.

\subsection{Projectivity and homological dimensions}

The following definitions shall be given in the case of left modules; the definitions in the cases of right modules and bimodules are similar, just use the following category isomorphisms: for unital $A, B$ we have

$$
\text { unmod- } A \simeq A^{\mathrm{op}} \text {-unmod } A \text {-unmod- } B \simeq\left(A \hat{\otimes} B^{\mathrm{op}}\right) \text {-unmod }
$$

Let $A$ be a unital $\hat{\otimes}$-algebra.

Definition 1.7. A complex of $A$ - $\hat{\otimes}$-modules $\{M, d\}$ is called admissible $\Longleftrightarrow$ it splits in the category LCS. A morphism of $A$ - $\hat{\otimes}$-modules $f: X \rightarrow Y$ is called admissible if it is one of the morphisms in an admissible complex.

Definition 1.8. An additive functor $F: A$ - $\bmod \rightarrow$ Lin is called exact $\Longleftrightarrow$ for every admissible complex $\{M, d\}$ the corresponding complex $\{F(M), F(d)\}$ in Lin is exact.

Definition 1.9. Suppose that $A$ and $B$ are unital $\hat{\otimes}$-algebras.

(1) A module $P \in A$-unmod is called projective $\Longleftrightarrow$ the functor $\operatorname{Hom}_{A}(P,-)$ is exact.

(2) A module $X \in A$-unmod is called free $\Longleftrightarrow X$ is isomorphic to $A \hat{\otimes} E$ for some $E \in \mathbf{L C S}$.

Now we consider the general, non-unital case. Let $A$ be a $\hat{\otimes}$-algebra. Any left $\hat{\otimes}$-module over an algebra $A$ can be viewed as a unital $\hat{\otimes}$-module over $A_{+}$, in other words, the following isomorphism of categories takes place:

$$
A \text {-mod } \cong A_{+} \text {-unmod, } A \text {-mod- } B \cong A_{+} \hat{\otimes} B_{+}^{\text {op }} \text {-unmod } .
$$

By using this isomorphism we can define projective and free modules in the non-unital case.

Definition 1.10. Suppose that $A$ and $B$ are $\hat{\otimes}$-algebras. 
(1) A module $P \in A$-mod is called projective $\Longleftrightarrow$ the module $P$ is projective in the category $A_{+}$-unmod

(2) A module $X \in A$-mod is called free $\Longleftrightarrow X$ is isomorphic to $A_{+} \hat{\otimes} E$ for some $E \in \mathbf{L C S}$.

As it turns out, there is no ambiguity, a unital module is projective in the sense of the Definition 1.9 if and only if it is projective in the sense of the Definition 1.10 ,

Definition 1.11. Let $X \in A$-mod. Suppose that $X$ can be included in a following admissible complex:

$$
0 \leftarrow X \stackrel{\varepsilon}{\leftarrow} P_{0} \stackrel{d_{0}}{\longleftarrow} P_{1} \stackrel{d_{1}}{\longleftarrow} \ldots \stackrel{d_{n-1}}{\longleftarrow} P_{n} \leftarrow 0 \leftarrow 0 \leftarrow \ldots,
$$

where every $P_{i}$ is a projective module. Then we will call the complex $\{P, d\}$, where

$$
\{P, d\}=0 \leftarrow P_{0} \stackrel{d_{0}}{\longleftarrow} P_{1} \stackrel{d_{1}}{\longleftarrow} \ldots \stackrel{d_{n-1}}{\longleftarrow} P_{n} \leftarrow 0,
$$

the projective resolution of $X$ of length $n$. By definition, the length of an unbounded resolution equals $\infty$.

This allows us to define the notion of a derived functor in the topological case, for example, see [Hel86, ch 3.3]. In particular, $\operatorname{Ext}_{A}^{k}(M, N)$ and $\operatorname{Tor}_{k}^{A}(M, N)$ are defined similarly to the purely algebraic situation.

Definition 1.12. Consider an arbitrary module $M \in A$-mod. Then following number is well-defined:

$$
\begin{aligned}
\operatorname{dh}_{A}(M) & =\min \left\{n \in \mathbb{Z}_{\geq 0}: \operatorname{Ext}_{A}^{n+1}(M, N)=0 \text { for every } N \in A \text {-mod }\right\}= \\
& =\{\text { the length of a shortest projective resolution of } M\} \in\{-\infty\} \cup[0, \infty] .
\end{aligned}
$$

It is called the projective (homological) dimension of $M$.

Definition 1.13. Let $A$ be a $\hat{\otimes}$-algebra. Then we can define the following invariants of $A$ :

$$
\begin{aligned}
& \operatorname{dgl}(A)=\sup \left\{\operatorname{dh}_{A}(M): M \in A \text {-mod }\right\} \text { - the left global dimension of } A . \\
& \operatorname{dgr}(A)=\sup \left\{\operatorname{dh}_{A}(M): M \in \bmod -A\right\}-\text { the right global dimension of } A .
\end{aligned}
$$

\subsection{Algebra of rapidly decreasing functions}

Recall the definition of the space of rapidly decreasing functions on $\mathbb{R}^{n}$.

Definition 1.14. For $n>0$ define the Fréchet space

$$
\mathscr{S}\left(\mathbb{R}^{n}\right):=\left\{f: \mathbb{R}^{n} \rightarrow \mathbb{C}:\|f\|_{k, l}=\sup _{x \in \mathbb{R}^{n}}\left|x^{k} D^{l}(f)\right|<\infty \text { for all } k, l \in \mathbb{Z}_{\geq 0}^{n}\right\}
$$

where $x^{k}=x_{1}^{k_{1}} \ldots x_{n}^{k_{n}}$ and $D^{l}(f)=\frac{\partial^{l_{1}}}{\partial x_{1}^{l_{1}}} \ldots \frac{\partial^{l_{n}}}{\partial x_{n}^{l_{n}}} f$. The topology on $\mathscr{S}\left(\mathbb{R}^{n}\right)$ is defined by the system $\left\{\|f\|_{k, l}: k, l \in \mathbb{Z}_{\geq 0}^{n}\right\}$.

There are two natural ways to define the multiplication on $\mathscr{S}\left(\mathbb{R}^{n}\right)$ :

$$
\begin{gathered}
(f \cdot g)(x)=f(x) g(x) \quad \text { (pointwise product) } \\
(f * g)(x)=\int_{\mathbb{R}^{n}} f(y) g(x-y) \mathrm{d} y . \quad \text { (convolution product) }
\end{gathered}
$$

The following theorem is well-known.

Theorem 1.1. Fix $n \in \mathbb{N}$.

(1) $\left(\mathscr{S}\left(\mathbb{R}^{n}\right), \cdot\right)$ is a Fréchet-Arens-Michael algebra. 
(2) The Fourier transform induces an isomorphism of Arens-Michael algebras

$$
\begin{gathered}
\mathcal{F}_{n}:\left(\mathscr{S}\left(\mathbb{R}^{n}\right), \cdot\right) \longrightarrow\left(\mathscr{S}\left(\mathbb{R}^{n}\right), *\right), \\
\mathcal{F}_{n}(f)(x)=\int_{\mathbb{R}^{n}} f(y) e^{-2 \pi i\langle x, y\rangle} \mathrm{d} y_{1} \ldots \mathrm{d} y_{n}
\end{gathered}
$$

Proof. (1) The proof is very similar to the proof that $C^{\infty}\left(\mathbb{R}^{n}\right)$ is a Fréchet-Arens-Michael algebra, which can be found in Mal86, Section 4.4.(2)].

(2) See [Fol99, Theorem 8.22, Corollary 8.28] for the proof.

From now on we will write $\mathscr{S}\left(\mathbb{R}^{n}\right)$ instead of $\left(\mathscr{S}\left(\mathbb{R}^{n}\right), \cdot\right)$ and $\mathscr{S}\left(\mathbb{R}^{n}\right)_{\text {conv }}$ instead of $\left(\mathscr{S}\left(\mathbb{R}^{n}\right)\right.$,*).

\section{$1.4 \widehat{\Omega}^{1}$-like admissible sequences for $\mathscr{S}(\mathbb{R})$}

In order to obtain the homological dimensions of $\mathscr{S}\left(\mathbb{R}^{n}\right)$ in the paper [OK84], Helemskii and Ogneva used a simple and natural $\widehat{\Omega}^{1}$-like admissible sequence for $\mathscr{S}(\mathbb{R})$. It was constructed using Hadamard's lemma.

Lemma 1.1 (Hadamard's lemma). Let $f \in \mathscr{S}\left(\mathbb{R}^{n}\right)$, such that $f\left(0, x_{2}, \ldots, x_{n}\right)=0$ for all $\left(x_{2}, \ldots, x_{n}\right) \in \mathbb{R}^{n-1}$. Then there exists a function $g \in \mathscr{S}\left(\mathbb{R}^{n}\right)$ such that

$$
f\left(x_{1}, \ldots, x_{n}\right)=x_{1} g\left(x_{1}, \ldots, x_{n}\right) .
$$

More generally, suppose that $f(x)=0$ on a hyperplane in $\mathbb{R}^{n}$ defined by the equation $a_{1} x_{1}+\cdots+a_{n} x_{n}=$ 0 . Then there exists $g \in \mathscr{S}\left(\mathbb{R}^{n}\right)$ such that

$$
f\left(x_{1}, \ldots, x_{n}\right)=\left(a_{1} x_{1}+\cdots+a_{n} x_{n}\right) g\left(x_{1}, \ldots, x_{n}\right) .
$$

Recall that $\mathscr{S}\left(\mathbb{R}^{2}\right)$ admits the following structure of a $\mathscr{S}(\mathbb{R})$ - $\hat{\otimes}$-bimodule:

$$
(\varphi \cdot f)(x, y)=\varphi(x) f(x, y), \quad(f \cdot \varphi)(x, y)=f(x, y) \varphi(y)
$$

for any $\varphi \in \mathscr{S}(\mathbb{R}), f \in \mathscr{S}\left(\mathbb{R}^{2}\right), x, y \in \mathbb{R}$.

The Theorem 1.1 gives a similar $\mathscr{S}(\mathbb{R})_{\text {conv- }} \hat{\otimes}$-bimodule structure on $\mathscr{S}\left(\mathbb{R}^{2}\right)_{\text {conv }}$.

Proposition 1.1 ([K84], Proposition 3). The following diagram is commutative, moreover, the rows of the diagram are short exact sequences of $\mathscr{S}(\mathbb{R})$ - $\hat{\otimes}$-bimodules which split in the categories $\mathscr{S}(\mathbb{R})-\bmod$ and $\bmod -\mathscr{S}(\mathbb{R})$ :

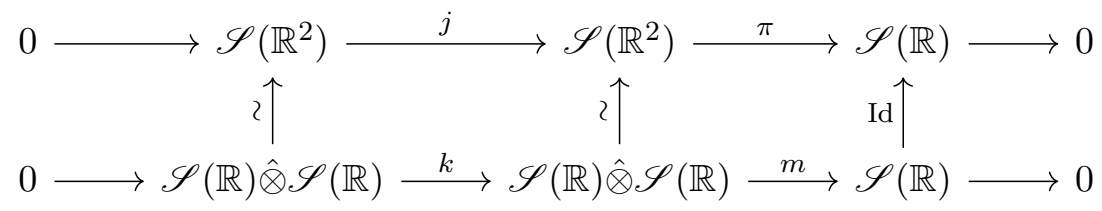

where

$$
\begin{aligned}
j(f)(x, y) & =(x-y) f(x, y) & & \text { for all } f \in \mathscr{S}\left(\mathbb{R}^{2}\right), \\
\pi(f)(x) & =f(x, x) & & \text { for all } f \in \mathscr{S}\left(\mathbb{R}^{2}\right) \\
k(f \otimes g) & =f x \otimes g-f \otimes g x & & \text { for all } f \in \mathscr{S}\left(\mathbb{R}^{2}\right), \\
m(f \otimes g) & =f g & & \text { for all } f \in \mathscr{S}\left(\mathbb{R}^{2}\right) .
\end{aligned}
$$

Let us restate the above proposition for $\mathscr{S}(\mathbb{R})_{\text {conv }}$. First of all, we will formulate a lemma which can be considered as the "Fourier dual" to Hadamard's lemma. 
Lemma 1.2. Let $f \in \mathscr{S}\left(\mathbb{R}^{n}\right)$ such that $\int_{\mathbb{R}} f\left(t, x_{2}, \ldots, x_{n}\right) \mathrm{d} t=0$ for any $\left(x_{2}, \ldots, x_{n}\right) \in \mathbb{R}^{n-1}$. Then there exists a function $g \in \mathscr{S}\left(\mathbb{R}^{n}\right)$ satisfying

$$
f\left(x_{1}, \ldots, x_{n}\right)=\frac{\partial}{\partial x_{1}} g\left(x_{1}, \ldots, x_{n}\right) .
$$

More generally, if there is a vector $v=\left(v_{1}, \ldots, v_{n}\right) \in \mathbb{R}^{n}$ such that the integral $\int_{\mathbb{R}} f(x+t v) \mathrm{d} t=0$ for any $x \in \mathbb{R}^{n}$, then there exists a function $g \in \mathscr{S}\left(\mathbb{R}^{n}\right)$ satisfying

$$
f\left(x_{1}, \ldots, x_{n}\right)=\left(\sum_{i=1}^{n} v_{i} \frac{\partial}{\partial x_{i}}\right) g\left(x_{1}, \ldots, x_{n}\right) .
$$

Proposition 1.2. The following diagram is commutative, moreover, the rows of the diagram are short exact sequences of $\mathscr{S}(\mathbb{R})_{\text {conv-}}-\hat{\otimes}$-bimodules which split in the categories $\mathscr{S}(\mathbb{R})_{\text {conv }}$-mod and $\bmod -\mathscr{S}(\mathbb{R})_{\text {conv }}$ :

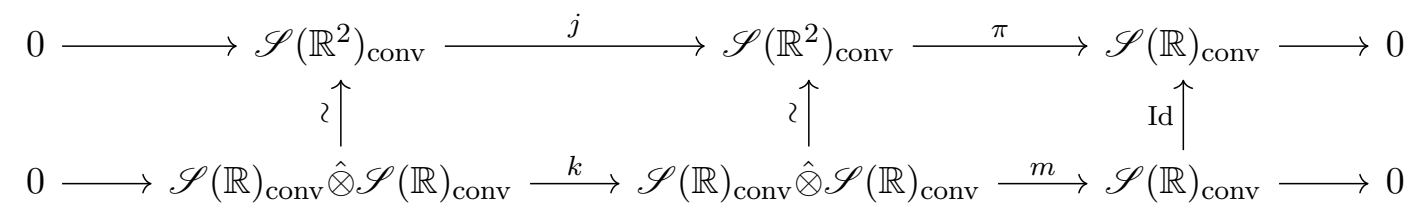

where

$$
\begin{aligned}
j(f)(x, y) & =\left(\frac{\partial}{\partial x}-\frac{\partial}{\partial y}\right) f(x, y) & & \text { for all } f \in \mathscr{S}\left(\mathbb{R}^{2}\right), \\
\pi(f)(x) & =\int_{\mathbb{R}} f(y, x-y) \mathrm{d} y & & \text { for all } f \in \mathscr{S}\left(\mathbb{R}^{2}\right) . \\
k(f \otimes g) & =f^{\prime} \otimes g-f \otimes g^{\prime} & & \text { for all } f \in \mathscr{S}\left(\mathbb{R}^{2}\right), \\
m(f \otimes g) & =f * g & & \text { for all } f \in \mathscr{S}\left(\mathbb{R}^{2}\right) .
\end{aligned}
$$

In the next section we will show that the diagram 3 can be generalized if we replace $\mathscr{S}(\mathbb{R})$ with smooth crossed products of Fréchet-Arens-Michael algebras by $\mathbb{R}$ and $\mathbb{T}$.

\section{$2 \widehat{\Omega}^{1}$-like admissible sequences for smooth crossed products}

\subsection{Smooth m-tempered actions and smooth crossed products}

Definition 2.1. Let $E$ be a Hausdorff topological vector space. For a function $f: \mathbb{R}^{n} \rightarrow E$ and $x \in \mathbb{R}$ we denote

$$
\frac{\partial f}{\partial x_{i}}(x):=\lim _{h \rightarrow 0} \frac{f\left(x_{1}, \ldots, x_{i}+h, \ldots, x_{n}\right)-f\left(x_{1}, \ldots, x_{i}, \ldots, x_{n}\right)}{h} .
$$

Definition 2.2. Let $X$ be a Fréchet space with topology, generated by a sequence of seminorms $\left\{\|\cdot\|_{m}: m \in \mathbb{N}\right\}$.

(1) The space $\mathscr{S}\left(\mathbb{T}^{n}, X\right):=C^{\infty}\left(\mathbb{T}^{n}, X\right)$ is a Fréchet space with respect to the system

$$
\left\{\|f\|_{k, m}=\sup _{x \in \mathbb{T}^{n}}\left\|D^{k}(f)(x)\right\|_{m}: k \in \mathbb{Z}_{\geq 0}^{n}, m \in \mathbb{N}\right\}
$$

(2) Define the following space:

$$
\mathscr{S}\left(\mathbb{R}^{n}, X\right)=\left\{f: \mathbb{R}^{n} \rightarrow X:\|f\|_{k, l, m}:=\sup _{x \in \mathbb{R}^{n}}\left\|x^{l} D^{k}(f)(x)\right\|_{m}<\infty \text { for all } k, l \in \mathbb{Z}_{\geq 0}^{n}, m \in \mathbb{N}\right\},
$$

where $D^{k}(f)=\frac{\partial^{k_{1}}}{\partial x_{i}^{k_{1}}} \ldots \frac{\partial^{k_{n}}}{\partial x_{i}^{k_{n}}} f$. (assuming $\mathbb{T}=\mathbb{R} / \mathbb{Z}$.) The topology on $\mathscr{S}\left(\mathbb{R}^{n}, X\right)$ is defined by the system $\left\{\|f\|_{k, l, m}: k, l \in \mathbb{Z}_{\geq 0}^{n}, m \in \mathbb{N}\right\}$.

The following proposition can be proven in the same way as in the [Mal86, Chapter 11.2]. 
Proposition 2.1. Let $A$ be a Fréchet space. Then the natural maps

$$
\begin{aligned}
\mathscr{S}\left(\mathbb{R}^{n}\right) \hat{\otimes} A \rightarrow \mathscr{S}\left(\mathbb{R}^{n}, A\right), \quad f \otimes a \mapsto(x \mapsto f(x) a), \\
\mathscr{S}\left(\mathbb{T}^{n}\right) \hat{\otimes} A \rightarrow \mathscr{S}\left(\mathbb{T}^{n}, A\right), \quad f \otimes a \mapsto(x \mapsto f(x) a) .
\end{aligned}
$$

are topological isomorphisms for $n \in \mathbb{N}$. As a corollary, we have

$$
\begin{aligned}
& \mathscr{S}\left(\mathbb{R}^{m}\right) \hat{\otimes} \mathscr{S}\left(\mathbb{R}^{n}\right) \cong \mathscr{S}\left(\mathbb{R}^{m}, \mathscr{S}\left(\mathbb{R}^{n}\right)\right) \cong \mathscr{S}\left(\mathbb{R}^{n+m}\right), \\
& \mathscr{S}\left(\mathbb{T}^{m}\right) \hat{\otimes} \mathscr{S}\left(\mathbb{T}^{n}\right) \cong \mathscr{S}\left(\mathbb{T}^{m}, \mathscr{S}\left(\mathbb{T}^{n}\right)\right) \cong \mathscr{S}\left(\mathbb{T}^{n+m}\right),
\end{aligned}
$$

This proposition gives us another way to differentiate and integrate vector-valued Schwartz functions.

Definition 2.3. Let $A$ be a Fréchet algebra. Then for $G=\mathbb{T}, \mathbb{R}$ we define the derivative $\frac{d}{d x}: \mathscr{S}(G, A) \rightarrow \mathscr{S}(G, A)$ and the integral $\int_{G}: \mathscr{S}(G, A) \rightarrow A$ using the universal property of the completed projective tensor product:

$$
\frac{d}{d x}(f \otimes a):=\left(\frac{d}{d x} f(x)\right) \otimes a, \quad \int_{G}(f \otimes a) d \mu:=\left(\int_{G} f d \mu\right) \otimes a .
$$

Definition 2.4. Let $A$ be a Fréchet-Arens-Michael algebra, and let $G=\mathbb{R}$ or $G=\mathbb{T}$. Then the action of $G$ on a $A$ is called:

(a) m-tempered, if there exists a generating family of submultiplicative seminorms $\left\{\|\cdot\|_{m}\right\}_{m \in \mathbb{N}}$ on $A$ such that for every $m \in \mathbb{N}$ there is a polynomial $p_{m}(x) \in \mathbb{R}[x]$, satisfying

$$
\left\|\alpha_{x}(a)\right\|_{m} \leq\left|p_{m}(x)\right|\|a\|_{m} \quad(a \in A, x \in G) .
$$

(b) $C^{\infty}$-m-tempered or smooth $m$-tempered, if the following conditions are satisfied:

(1) for every $a \in A$ the function

$$
\alpha_{x}(a): G \longrightarrow A, \quad x \mapsto \alpha_{x}(a),
$$

is $C^{\infty}$-differentiable,

(2) there exists a generating family of submultiplicative seminorms $\left\{\|\cdot\|_{m}\right\}_{m \in \mathbb{N}}$ on $A$ such that for any $k \geq 0$ and $m>0$ there exists a polynomial $p_{k, m} \in \mathbb{R}[x]$, satisfying

$$
\left\|\alpha_{x}^{(k)}(a)\right\|_{m} \leq\left|p_{k, m}(x)\right|\|a\|_{m} \quad(k \in \mathbb{N}, x \in G, a \in A) .
$$

The following theorem can be viewed as a definition of smooth crossed products.

Theorem 2.1 ([Sch93], Theorem 3.1.7). Let $A$ be a Fréchet-Arens-Michael algebra with an $m$-tempered action of one of the groups $G=\mathbb{R}$ or $G=\mathbb{T}$. Then the space $\mathscr{S}(G, A)$ endowed with the following multiplication:

$$
\left(f *_{\alpha} g\right)(x)=\int_{G} f(y) \alpha_{y}(g(x-y)) d y
$$

becomes a Fréchet-Arens-Michael algebra.

When $G=\mathbb{R}$, we will denote this algebra by $\mathscr{S}(\mathbb{R}, A ; \alpha)$, and in the case $G=\mathbb{T}$ we will write $C^{\infty}(\mathbb{T}, A ; \alpha)$.

Remark. If $\alpha$ is the trivial action, then $\mathscr{S}(G, A ; \alpha)=\mathscr{S}(G, A)$ with the usual convolution product.

Proposition 2.2. Let $A$ be a Fréchet-Arens-Michael algebra. Consider an action $\alpha: \mathbb{R} \rightarrow \operatorname{Aut}(A)$. Then $\alpha$ is a smooth $m$-tempered action if and only if the following holds: 
1. the derivative $\alpha_{x}^{\prime}(a)$ exists at $x=0$ for every $a \in A$, and, as a corollary, derivatives all of orders at zero exist.

2. there exists a generating family of submultiplicative seminorms $\left\{\|\cdot\|_{m}\right\}_{m \in \mathbb{N}}$ on $A$ such that for every $m \in \mathbb{N}$ and $k \in \mathbb{N}$ there exist polynomials $p_{m}(x) \in \mathbb{R}[x]$ and $C_{k, m}>0$, satisfying

$$
\left\|\alpha_{x}(a)\right\|_{m} \leq\left|p_{m}(x)\right|\|a\|_{m}, \quad\left\|\alpha_{0}^{(k)}(a)\right\|_{m} \leq C_{k, m}\|a\|_{m}
$$

for every $a \in A, x \in \mathbb{R}$.

Proof. $(\Rightarrow)$ If $\alpha$ is $C^{\infty}-m$-tempered, choose the seminorms $\|\cdot\|_{m}$ and the polynomials $p_{m, k}(x)$ as in the Definition 2.4, and set

$$
p_{m}(x)=p_{0, m}(x), \quad C_{k, m}=p_{k, m}(0) .
$$

$(\Leftarrow)$ Notice that

$$
\alpha_{x}^{\prime}(a)=\lim _{h \rightarrow 0} \frac{\alpha_{x+h}(a)-\alpha_{x}(a)}{h}=\alpha_{x}\left(\lim _{h \rightarrow 0} \frac{\alpha_{h}(a)-a}{h}\right)=\alpha_{x}\left(\alpha_{0}^{\prime}(a)\right) \quad(a \in A) .
$$

Therefore,

$$
\alpha_{x}^{(k)}(a)=\alpha_{x}^{(k-1)}\left(\alpha_{0}^{\prime}(a)\right)=\alpha_{x}^{(k-2)}\left(\alpha_{0}^{\prime}\left(\alpha_{0}^{\prime}(a)\right)\right)=\cdots=\alpha_{x}(\underbrace{\alpha_{0}^{\prime}\left(\ldots\left(\alpha_{0}^{\prime}(a)\right)\right)}_{k \text { times }})
$$

However,

$$
\alpha_{0}^{\prime}\left(\alpha_{0}^{\prime}(x)\right)=\lim _{h \rightarrow 0} \frac{\alpha_{h}\left(\alpha_{0}^{\prime}(x)\right)-\alpha_{0}^{\prime}(x)}{h}=\lim _{h \rightarrow 0} \frac{\alpha_{h}^{\prime}(x)-\alpha_{0}^{\prime}(x)}{h}=\alpha_{0}^{\prime \prime}(x) .
$$

By induction we obtain the following equality:

$$
\alpha_{x}^{(k)}(a)=\alpha_{x}\left(\alpha_{0}^{(k)}(a)\right)
$$

for every $a \in A, x \in \mathbb{R}, k \in \mathbb{Z}_{\geq 0}$.

As an immediate corollary, $\alpha_{x}(a) \in C^{\infty}(\mathbb{R}, A)$ for every $a \in A$. This also implies that

$$
\left\|\alpha_{x}^{(k)}(a)\right\|_{m}=\left\|\alpha_{x}\left(\alpha_{0}^{(k)}(x)\right)\right\|_{m} \leq\left|p_{m}(x)\right|\left\|\alpha_{0}^{(k)}(a)\right\|_{m} \leq\left|p_{m}(x)\right| C_{k, m}\|a\|_{m} .
$$

Now set $p_{k, m}(x)=C_{k, m} p_{m}(x)$.

The proposition can be restated for $G=\mathbb{T}$ :

Proposition 2.3. Let $A$ be a Fréchet-Arens-Michael algebra. Consider an action $\alpha: \mathbb{T} \rightarrow \operatorname{Aut}(A)$. Then $\alpha$ is a smooth $m$-tempered action if and only if the following holds:

1. the derivative $\alpha_{x}^{\prime}(a)$ exists at $x=0$ for every $a \in A$, and, as a corollary, derivatives all of orders at zero exist.

2. there exists a generating family of submultiplicative seminorms $\left\{\|\cdot\|_{m}\right\}_{m \in \mathbb{N}}$ on $A$ such that for every $m \in \mathbb{N}$ and $k \in \mathbb{N}$ there exist $C_{m}, C_{k, m}>0$, satisfying

$$
\left\|\alpha_{x}(a)\right\|_{m} \leq C_{m}\|a\|_{m}, \quad\left\|\alpha_{0}^{(k)}(a)\right\|_{m} \leq C_{k, m}\|a\|_{m}
$$

for every $a \in A, x \in \mathbb{T}$.

Proof. The proof is the same as in the previous proposition, we only need keep in mind that

$$
\left|p_{k, m}(x)\right| \leq \sup _{x \in \mathbb{T}}\left|p_{k, m}(x)\right|<\infty .
$$




\subsection{Explicit construction}

Remark. In this subsection we only treat the case $G=\mathbb{R}$ here, the case $G=\mathbb{T}$ can be dealt with in the same way.

Definition 2.5. A $\hat{\otimes}$-algebra $A$ is called self-induced, if the multiplication map $m: A \hat{\otimes}_{A} A \longrightarrow A$ is a $A$ - $\hat{\otimes}$-bimodule isomorphism.

Until the end of this section, $A$ will denote a self-induced Fréchet-Arens-Michael algebra. Also let $\alpha$ denote a smooth $m$-tempered $\mathbb{R}$-action of $A$.

In this subsection we will construct a $\hat{\Omega}_{A}^{1}$-like admissible sequence for $\mathscr{S}(\mathbb{R}, A ; \alpha)$.

Proposition 2.4. For any $F \in \mathscr{S}(\mathbb{R}, A)$ define $T(F)(x)=\alpha_{x}(F(x))$. Then the following statements hold:

1. The mapping $T$ is a well-defined continuous linear map $T: \mathscr{S}(\mathbb{R}, A) \rightarrow \mathscr{S}(\mathbb{R}, A)$,

2. Moreover, $T$ is invertible, with the inverse, defined for every $F \in \mathscr{S}(\mathbb{R}, A)$ as follows:

$$
T^{-1}(F)(x)=\alpha_{-x}(F(x)) .
$$

In particular, we have

$$
\left(T \circ \frac{\mathrm{d}}{\mathrm{d} x} \circ T^{-1}\right)(F)(x)=F^{\prime}(x)-\alpha_{0}^{\prime}(F(x))
$$

for any $F \in \mathscr{S}(\mathbb{R}, A)$.

3. For any $F, G \in \mathscr{S}(\mathbb{R}, A ; \alpha)$ we have

$$
F^{\prime} *_{\alpha} T(G)=F *_{\alpha} T\left(G^{\prime}\right) .
$$

This equality is equivalent to

$$
F^{\prime} *_{\alpha} G=F *_{\alpha}\left(T \circ \frac{\mathrm{d}}{\mathrm{d} x} \circ T^{-1}\right)(G) .
$$

Proof.

1. Let us write down the derivative of $\alpha_{x}(F(x))$ :

$$
\begin{aligned}
\frac{\mathrm{d}}{\mathrm{d} x}\left(\alpha_{x}(F(x))\right) & =\lim _{h \rightarrow 0} \frac{\alpha_{x+h}(F(x+h))-\alpha_{x}(F(x))}{h}= \\
& =\alpha_{x}\left(\lim _{h \rightarrow 0} \frac{\alpha_{h}\left(F(x)+F^{\prime}(x) h+o(h)\right)-F(x)}{h}\right)= \\
& =\alpha_{x}\left(\alpha_{0}^{\prime}(F(x))+F^{\prime}(x)\right)=\alpha_{x}^{\prime}(F(x))+\alpha_{x}\left(F^{\prime}(x)\right) .
\end{aligned}
$$

It is easily seen that

$$
\frac{\mathrm{d}^{k}}{\mathrm{~d} x^{k}}\left(\alpha_{x}(F(x))\right)=\sum_{i=0}^{k}\left(\begin{array}{l}
n \\
k
\end{array}\right) \alpha_{x}^{(i)}\left(F^{(k-i)}(x)\right) .
$$

Now fix a generating system of seminorms on $A$ which satisfies the conditions of the Proposition 2.2. Let us show that $\alpha_{x}^{(m)}(F(x))$ lies in $\mathscr{S}(\mathbb{R}, A)$ for any $F \in \mathscr{S}(\mathbb{R}, A)$ and $m \geq 0$ :

$$
\begin{aligned}
& \|T(F)\|_{k, l, m}=\sup _{x \in \mathbb{R}}\left\|x^{l} \frac{\mathrm{d}^{k}}{\mathrm{~d} x^{k}}\left(\alpha_{x}(F(x))\right)\right\|_{m} \leq \sum_{i=0}^{k}\left(\begin{array}{c}
k \\
i
\end{array}\right) \sup _{x \in \mathbb{R}}\left\|\alpha_{x}^{(i)}\left(F^{(k-i)}(x)\right)\right\| \leq \\
& \leq \sum_{i=0}^{k}\left(\begin{array}{c}
k \\
i
\end{array}\right) \sup _{x \in \mathbb{R}}\left(\left|p_{i, m}(x)\right|\left\|F^{(k-i)}(x)\right\|_{m}\right)<\infty .
\end{aligned}
$$


2. Notice that the same argument shows works for $T^{-1}$, as well. As for the equality, notice that

$$
\frac{\mathrm{d}}{\mathrm{d} x}\left(\alpha_{-x}(F(x))\right)=-\alpha_{-x}^{\prime}(F(x))+\alpha_{-x}\left(F^{\prime}(x)\right),
$$

so we have

$$
T\left(\frac{\mathrm{d}}{\mathrm{d} x}\left(\alpha_{-x}(F(x))\right)\right)=-\alpha_{x}\left(\alpha_{0}^{\prime}(F(x))\right)+\left(F^{\prime}(x)\right) \stackrel{\underline{\underline{5}}}{=}-\alpha_{0}^{\prime}(F(x))+F^{\prime}(x)
$$

3. This is equivalent to

$$
\begin{aligned}
\int_{\mathbb{R}} F^{\prime}(y) \alpha_{y}(T G(x-y)) \mathrm{d} y & =\int_{\mathbb{R}} F(y) \alpha_{y}\left(T G^{\prime}(x-y)\right) \mathrm{d} y \Leftrightarrow \\
\Leftrightarrow \int_{\mathbb{R}} F^{\prime}(y) \alpha_{x}(G(x-y)) \mathrm{d} y & =\int_{\mathbb{R}} F(y) \alpha_{x}\left(G^{\prime}(x-y)\right) \mathrm{d} y \Leftrightarrow \\
\Leftrightarrow \int_{\mathbb{R}} \frac{\mathrm{d}}{\mathrm{d} y}(F(y)) \alpha_{x}(G(x-y)) \mathrm{d} y & =-\int_{\mathbb{R}} F(y) \frac{\mathrm{d}}{\mathrm{d} y}\left(\alpha_{x}(G(x-y))\right) \mathrm{d} y \Leftrightarrow \\
\Leftrightarrow \int_{\mathbb{R}} \frac{\mathrm{d}}{\mathrm{d} y}(F(y)) \alpha_{x}(G(x-y)) \mathrm{d} y & +\int_{\mathbb{R}} F(y) \frac{\mathrm{d}}{\mathrm{d} y}\left(\alpha_{x}(G(x-y))\right) \mathrm{d} y=0 \quad \text { (integration by parts) }
\end{aligned}
$$

Let $\mathscr{S}(\mathbb{R}, A ; \alpha)_{\alpha}=: S_{\alpha}$ denote the $\mathscr{S}(\mathbb{R}, A ; \alpha)$ - $\hat{\otimes}$-bimodule and $A$ - $\hat{\otimes}$-bimodule, which coincides with $\mathscr{S}(\mathbb{R}, A)$ as a LCS, and the bimodule actions are given below:

$$
\begin{aligned}
& (F \circ a)(x)=F(x) \alpha_{x}(a), \quad a \circ F(x)=a F(x) \quad \text { for any } a \in A, F \in S_{\alpha} \\
& (F \circ G)(x)=\left(F *_{\alpha} G\right)(x), \quad(G \circ F)(x)=\left(G *_{\alpha} F\right)(x) \quad \text { for any } F, G \in S_{\alpha}
\end{aligned}
$$

\section{Proposition 2.5.}

1. For any $F \in \mathscr{S}(\mathbb{R}, A)$ and $a \in A$ the functions $F \circ a$ and $a \circ F$ belong to $\mathscr{S}(\mathbb{R}, A)$. As a corollary, $S_{\alpha}$ is well-defined.

2. The following equalities take place:

$$
\begin{gathered}
(F \circ a)^{\prime}=\left(F^{\prime} \circ a\right)+\left(F \circ \alpha_{0}^{\prime}(a)\right), \\
\left(T \circ \frac{\mathrm{d}}{\mathrm{d} x} \circ T^{-1}\right)(F \circ a)=\left(\left(T \circ \frac{\mathrm{d}}{\mathrm{d} x} \circ T^{-1}\right) F\right) \circ a, \\
\left(T \circ \frac{\mathrm{d}}{\mathrm{d} x} \circ T^{-1}\right)(a \circ F)=a \circ\left(T \circ \frac{\mathrm{d}}{\mathrm{d} x} \circ T^{-1}\right)(F)-\alpha_{0}^{\prime}(a) \circ F .
\end{gathered}
$$

Proof.

1. The argument for $a \circ F$ is pretty much trivial, we only need to check that $F \circ a \in \mathscr{S}(\mathbb{R}, A)$. Fix a generating system of seminorms $\left\{\|\cdot\|_{m}\right\}$ on $A$, satisfying the conditions of the Proposition 2.2 , Notice that for every $k, l \in \mathbb{Z}_{\geq 0}$ and $m \in \mathbb{N}$ we have

$$
\begin{aligned}
\|F \circ a\|_{k, l, m} & =\sup _{x \in \mathbb{R}}\left\|x^{l} \frac{\mathrm{d}^{k}}{\mathrm{~d} x^{k}}(F \circ a)(x)\right\|_{m}=\sup _{x \in \mathbb{R}}\left\|\sum_{i=0}^{k} x^{l}\left(\begin{array}{c}
k \\
i
\end{array}\right) F^{(i)}(x) \alpha_{0}^{(k-i)}(a)\right\|_{m} \leq \\
& \leq \sum_{i=0}^{k}\left(\begin{array}{c}
k \\
i
\end{array}\right)\left|x^{l}\right|\left\|F^{(i)}(x)\right\|_{m}\left\|\alpha_{0}^{(k-i)}(a)\right\|_{m} \leq\|a\|_{m} \sum_{i=0}^{k}\left(\begin{array}{c}
k \\
i
\end{array}\right) C_{k-i, m}\left|x^{l}\right|\left\|F^{(i)}(x)\right\|_{m}= \\
& =\|a\|_{m} \sum_{i=0}^{k}\left(\begin{array}{c}
k \\
i
\end{array}\right) C_{k-i, m}\|F\|_{i, l, m}<\infty .
\end{aligned}
$$


2. Checking these equalities is pretty straightforward:

$$
\begin{gathered}
(F \circ a)^{\prime}(x)=F(x) \alpha_{x}^{\prime}(a)+F^{\prime}(x) \alpha_{x}(a) \stackrel{\text { 吕 }}{=} F(x) \alpha_{x}\left(\alpha_{0}^{\prime}(a)\right)+F^{\prime}(x) \alpha_{x}(a)=\left(F^{\prime} \circ a\right)(x)+\left(F \circ \alpha_{0}^{\prime}(a)\right)(x), \\
\left(T \circ \frac{\mathrm{d}}{\mathrm{d} x} \circ T^{-1}\right)(F \circ a)=\left(\left(T \circ \frac{\mathrm{d}}{\mathrm{d} x}\right)\left(T^{-1} F\right) \circ a\right)=\left(\left(T \circ \frac{\mathrm{d}}{\mathrm{d} x} \circ T^{-1}\right) F\right) \circ a . \\
\left(T \circ \frac{\mathrm{d}}{\mathrm{d} x} \circ T^{-1}\right)(a \circ F)(x)=\left(T \circ \frac{\mathrm{d}}{\mathrm{d} x}\right)\left(\alpha_{-x}(a)\left(T^{-1} F\right)(x)\right)= \\
=T\left(\alpha_{-x}(a)\left(T^{-1} F\right)^{\prime}(x)-\alpha_{-x}^{\prime}(a)\left(T^{-1} F\right)(x)\right)=\left(a \circ\left(T \circ \frac{\mathrm{d}}{\mathrm{d} x} \circ T^{-1}\right)(F)-\alpha_{0}^{\prime}(a) \circ F\right)(x) .
\end{gathered}
$$

Lemma 2.1. The bimodule $S_{\alpha}$ belongs to the categories $\mathscr{S}(\mathbb{R}, A ; \alpha)$-mod- $A(\mathbf{F r})$ and $A$-mod- $\mathscr{S}(\mathbb{R}, A ; \alpha)(\mathbf{F r})$. In particular, the following $\mathscr{S}(\mathbb{R}, A ; \alpha)$ - $\hat{\otimes}$-bimodule structure on $S_{\alpha} \hat{\otimes}_{A} S_{\alpha}$ is well-defined:

$$
H \circ(F \otimes G)=\left(H *_{\alpha} F\right) \otimes G, \quad(F \otimes G) \circ H=F \otimes\left(G *_{\alpha} H\right)
$$

for any $F, G, H \in \mathscr{S}(\mathbb{R}, A)$.

Proof. We only need to prove that $(H \circ F) \circ a=H \circ(F \circ a)$ and $(a \circ F) \circ H=a \circ(F \circ H)$ for any $a \in A ; F, H \in S_{\alpha}$.

$$
\begin{gathered}
H \circ(F \circ a)(x)=\int_{\mathbb{R}} H(y) \alpha_{y}(F(x-y)) \alpha_{x}(a) \mathrm{d} y=(H \circ F) \circ a(x), \\
(a \circ F) \circ H(x)=\int_{\mathbb{R}} a F(y) \alpha_{y}(H(x-y)) \mathrm{d} y=a \circ(H \circ F)(x) .
\end{gathered}
$$

It is also easy to see that $S_{\alpha} \hat{\otimes}_{A} S_{\alpha}$ is a well-defined $A$ - $\hat{\otimes}$-bimodule.

Lemma 2.2. Define the following maps:

$$
\begin{array}{ll}
m: S_{\alpha} \hat{\otimes}_{A} S_{\alpha} \longrightarrow S_{\alpha}, & m(F \otimes G)=F *_{\alpha} G, \\
j: S_{\alpha} \hat{\otimes}_{A} S_{\alpha} \longrightarrow S_{\alpha} \hat{\otimes}_{A} S_{\alpha}, & j(F \otimes G)=F^{\prime} \otimes G-F \otimes\left(T \circ \frac{\mathrm{d}}{\mathrm{d} x} \circ T^{-1}\right) G .
\end{array}
$$

These maps are well-defined $\mathscr{S}(\mathbb{R}, A ; \alpha)$ - $\hat{\otimes}$-bimodule and $A$ - $\hat{\otimes}$-bimodule homomorphisms.

Proof. First of all, let us prove that $m$ and $j$ are well-defined:

$$
\begin{gathered}
m(F \circ a \otimes G)(x)=\int_{\mathbb{R}} F(y) \alpha_{y}(a) \alpha_{y}(G(x-y)) \mathrm{d} y=\int_{\mathbb{R}} F(y) \alpha_{y}(a G(x-y)) \mathrm{d} y=m(F \otimes a \circ G)(x, y) . \\
j(F \circ a \otimes G)=(F \circ a)^{\prime} \otimes G-(F \circ a) \otimes\left(T \circ \frac{\mathrm{d}}{\mathrm{d} x} \circ T^{-1}\right)(G) \stackrel{\underline{\underline{9}}}{ } \\
\underline{\underline{\underline{9}}}\left(F^{\prime} \circ a\right) \otimes G+\left(F \circ \alpha_{0}^{\prime}(a)\right) \otimes G-(F \circ a) \otimes\left(T \circ \frac{\mathrm{d}}{\mathrm{d} x} \circ T^{-1}\right)(G)= \\
=F^{\prime} \otimes a \circ G+F \otimes \alpha_{0}^{\prime}(a) \circ G-F \otimes a \circ\left(T \circ \frac{\mathrm{d}}{\mathrm{d} x} \circ T^{-1}\right)(G) . \\
j(F \otimes a \circ G)=F^{\prime} \otimes a \circ G-F(x) \otimes\left(T \circ \frac{\mathrm{d}}{\mathrm{d} x} \circ T^{-1}\right)(a \circ G) \stackrel{11}{=} \\
\underline{\underline{\underline{11}}} F^{\prime} \otimes a \circ G-F \otimes a \circ\left(T \circ \frac{\mathrm{d}}{\mathrm{d} x} \circ T^{-1}\right)(G)+F \otimes \alpha_{0}^{\prime}(a) \circ G=j(F \circ a \otimes G) .
\end{gathered}
$$

The algebra $\mathscr{S}(\mathbb{R}, A ; \alpha)$ is associative, therefore, $m$ is a $\mathscr{S}(\mathbb{R}, A ; \alpha)$ - $\hat{\otimes}$-bimodule homomorphism. 
It is relatively easy to show that $j$ is a left $\mathscr{S}(\mathbb{R}, A ; \alpha)$ - $\hat{\otimes}$-module homomorphism:

$$
\begin{aligned}
j\left(\left(H *_{\alpha} F\right) \otimes G\right) & =\left(H *_{\alpha} F\right)^{\prime} \otimes G-\left(H *_{\alpha} F\right) \otimes\left(T \circ \frac{\mathrm{d}}{\mathrm{d} x} \circ T^{-1}\right)(G)= \\
& =\left(H *_{\alpha} F^{\prime}\right) \otimes G-\left(H *_{\alpha} F\right) \otimes\left(T \circ \frac{\mathrm{d}}{\mathrm{d} x} \circ T^{-1}\right)(G)=H *_{\alpha} j(F \otimes G) .
\end{aligned}
$$

And it is slightly more difficult to show that it is a right $\mathscr{S}(\mathbb{R}, A ; \alpha)$ - $\hat{\otimes}$-module homomorphism.

$$
\begin{gathered}
j\left(F \otimes\left(G *_{\alpha} H\right)\right)=F^{\prime} \otimes\left(G *_{\alpha} H\right)-F \otimes T\left(\left(T^{-1}(G * H)\right)^{\prime}\right) \\
T^{-1}(G * H)(x)=\int_{\mathbb{R}} \alpha_{-x}(G(y)) \alpha_{-x+y}(H(x-y)) \mathrm{d} y=\int_{\mathbb{R}} \alpha_{-x}(G(y+x)) \alpha_{y}(H(-y)) \mathrm{d} y \\
\left(T^{-1}(G * H)\right)^{\prime}(x)=\int_{\mathbb{R}} \frac{\mathrm{d}}{\mathrm{d} x}\left(\alpha_{-x}(G(y+x))\right) \alpha_{y}(H(-y)) \mathrm{d} y \underline{\underline{\underline{8}}} \\
\underline{\underline{\underline{8}}} \int_{\mathbb{R}}\left(\alpha_{-x}\left(G^{\prime}(x+y)\right)-\alpha_{-x}^{\prime}(G(x+y))\right) \alpha_{y}(H(-y)) \mathrm{d} y . \\
T\left(\left(T^{-1}(G * H)\right)^{\prime}\right)(x)=\int_{\mathbb{R}}\left(G^{\prime}(x+y)-\alpha_{x}\left(\alpha_{-x}^{\prime}(G(x+y))\right)\right) \alpha_{x+y}(H(-y)) \mathrm{d} y \underline{\underline{\underline{5}}} \\
\underline{\underline{\underline{5}}} \int_{\mathbb{R}}\left(G^{\prime}(x+y)-\alpha_{0}^{\prime}(G(x+y))\right) \alpha_{x+y}(H(-y)) \mathrm{d} y= \\
=\int_{\mathbb{R}}\left(G^{\prime}(y)-\alpha_{0}^{\prime}(G(y))\right) \alpha_{y}(H(x-y)) \mathrm{d} y \underline{\underline{\underline{6}}}\left(\left(T \circ \frac{\mathrm{d}}{\mathrm{d} x} \circ T^{-1}\right)(G) *_{\alpha} H\right)(x) .
\end{gathered}
$$

Therefore, we have

$$
j\left(F \otimes\left(G *_{\alpha} H\right)\right)=F^{\prime} \otimes\left(G *_{\alpha} H\right)-F \otimes\left(T \circ \frac{\mathrm{d}}{\mathrm{d} x} \circ T^{-1}\right)(G) *_{\alpha} H=j(F \otimes G) *_{\alpha} H .
$$

Now let us check that $j$ and $m$ are $A$ - $\hat{\otimes}$-bimodule homomorphisms:

$$
\begin{array}{r}
m(a \circ F \otimes G)(x)=\int_{R} a F(y) \alpha_{y}(G(x-y)) \mathrm{d} y=(a \circ m(F \otimes G))(x) \\
m(F \otimes G \circ a)(x)=\int_{R} F(y) \alpha_{y}(G(x-y)) \alpha_{x}(a) \mathrm{d} y=(m(F \otimes G) \circ a)(x) \\
j(a \circ F \otimes G)=(a \circ F)^{\prime} \otimes G-a \circ F \otimes\left(T \circ \frac{\mathrm{d}}{\mathrm{d} x} \circ T^{-1}\right)(G)=a \circ j(F \otimes G) \\
j(F \otimes G \circ a)=F^{\prime} \otimes G \circ a-F \otimes\left(T \circ \frac{\mathrm{d}}{\mathrm{d} x} \circ T^{-1}\right)(G \circ a) \stackrel{10}{=} \\
\underline{\underline{\underline{10}}} F^{\prime} \otimes G \circ a-F(x) \otimes\left(T \circ \frac{\mathrm{d}}{\mathrm{d} x} \circ T^{-1}\right)(G) \circ a=j(F \otimes G) \circ a .
\end{array}
$$

As a corollary from the Proposition 2.4 we have $m \circ j=0$.

Proposition 2.6. The tensor product $S_{\alpha} \hat{\otimes}_{A} S_{\alpha}$ is isomorphic to $\mathscr{S}\left(\mathbb{R}^{2}, A\right)$ as a locally convex space:

$$
I_{1}: S_{\alpha} \hat{\otimes}_{A} S_{\alpha} \longrightarrow \mathscr{S}\left(\mathbb{R}^{2}, A\right), \quad I_{1}(F \otimes G)(x, y)=\alpha_{-x}(F(x)) G(y) .
$$

Proof. First of all, we can replace $\mathscr{S}\left(\mathbb{R}^{2}, A\right)$ with $\mathscr{S}\left(\mathbb{R}^{2}, A \hat{\otimes}_{A} A\right)$, because $A$ is isomorphic to $A \hat{\otimes}_{A} A$ as a locally convex space. This is precisely where we use the fact that $A$ is a self-induced algebra.

Let $X$ be a complete LCS and consider a continuous $A$-balanced map $Q: S_{\alpha} \times S_{\alpha} \longrightarrow X$. Define the map

$$
\widetilde{Q}: \mathscr{S}\left(\mathbb{R}^{2}, A \hat{\otimes}_{A} A\right) \longrightarrow X, \quad \widetilde{Q}(f(x) g(y) a \otimes b)=I_{1}\left(f(x) \alpha_{x}(a), g(x) b\right)
$$


This map is a well-defined continuous linear map, because $I_{1}$ is $A$-balanced and the linear span of $\{f(x) g(y) a \otimes b: f, g \in \mathscr{S}(\mathbb{R}), a, b \in A\}$ is dense in $\mathscr{S}\left(\mathbb{R}^{2}, A \hat{\otimes}_{A} A\right)$. From the construction of $\widetilde{Q}$ it follows that the following diagram is commutative:

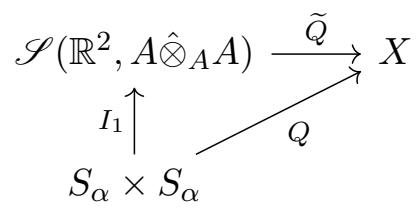

Moreover, $\widetilde{Q}$ is a unique mapping which makes this diagram commute.

Therefore, the isomorphism $I_{1}$ induces the structure of $S_{\alpha}$-module on $\mathscr{S}\left(\mathbb{R}^{2}, A\right)$, which we will denote by $\mathscr{S}\left(\mathbb{R}^{2}, A\right)_{\alpha}$. Let us describe the action of $\mathscr{S}(\mathbb{R}, A ; \alpha)$ and $A$ on $\mathscr{S}\left(\mathbb{R}^{2}, A\right)_{\alpha}$ explicitly.

Lemma 2.3. The algebra $A$ acts on $\mathscr{S}\left(\mathbb{R}^{2}, A\right)_{\alpha}$ as follows:

$$
\begin{array}{ll}
(a \circ F)(x, y)=\alpha_{-x}(a) F(x, y) & \text { for any } F \in \mathscr{S}\left(\mathbb{R}^{2}, A\right)_{\alpha}, a \in A \\
(F \circ a)(x, y)=F(x, y) \alpha_{y}(a) & \text { for any } F \in \mathscr{S}\left(\mathbb{R}^{2}, A\right)_{\alpha}, a \in A
\end{array}
$$

The algebra $\mathscr{S}(\mathbb{R}, A ; \alpha)$ acts on $\mathscr{S}\left(\mathbb{R}^{2}, A\right)_{\alpha}$ as follows:

$$
\begin{array}{ll}
(H \circ F)(x, y)=\int_{\mathbb{R}} \alpha_{-x}(H(z)) F(x-z, y) \mathrm{d} z & \text { for any } F \in \mathscr{S}\left(\mathbb{R}^{2}, A\right)_{\alpha}, H \in \mathscr{S}(\mathbb{R}, A ; \alpha) \\
(F \circ H)(x, y)=\int_{\mathbb{R}} F(x, z) \alpha_{z}(H(y-z)) \mathrm{d} z & \text { for any } F \in \mathscr{S}\left(\mathbb{R}^{2}, A\right)_{\alpha}, H \in \mathscr{S}(\mathbb{R}, A ; \alpha) .
\end{array}
$$

Proof. In all cases we will check every relation on a dense subset, then we will use the continuity arguments to finish the proof. Let $F(x, y)=G_{1}(x) G_{2}(y)$.

$$
\begin{aligned}
& (a \circ F)(x, y)=I_{1}\left(a \circ T G_{1} \otimes G_{2}\right)(x, y)=\alpha_{-x}(a) G_{1}(x) G_{2}(y)=\alpha_{-x}(a) F(x, y) \\
& (F \circ a)(x, y)=I_{1}\left(T G_{1} \otimes G_{2} \circ a\right)(x, y)=G_{1}(x) G_{2}(y) \alpha_{y}(a)=F(x, y) \alpha_{y}(a) \\
& (H \circ F)(x, y)=I_{1}\left(\int_{\mathbb{R}} H(z) \alpha_{z}\left(T G_{1}(x-z)\right) \mathrm{d} z \otimes G_{2}(x)\right) \otimes G_{2}(x)= \\
& =I_{1}\left(\int_{\mathbb{R}} H(z) \alpha_{x}\left(G_{1}(x-z)\right) \mathrm{d} z \otimes G_{2}(x)\right)=\left(\int_{\mathbb{R}} \alpha_{-x}(H(z))\left(G_{1}(x-z)\right) G_{2}(y) \mathrm{d} z\right)= \\
& =\int_{\mathbb{R}} \alpha_{-x}(H(z)) F(x-z, y) \mathrm{d} z . \\
& (F \circ H)(x, y)=I_{1}\left(T G_{1}(x) \otimes \int_{\mathbb{R}} G_{2}(z) \alpha_{z}(H(x-z)) \mathrm{d} z\right)=\int_{\mathbb{R}} G_{1}(x) G_{2}(z) \alpha_{z}(H(y-z)) \mathrm{d} z= \\
& =\int_{\mathbb{R}} F(x, z) \alpha_{z}(H(y-z)) \mathrm{d} z .
\end{aligned}
$$

Now we want to construct the right inverse maps to $m$ and $j$. First of all, let us describe the action of these maps on $\mathscr{S}\left(\mathbb{R}^{2}, A\right)$.

Lemma 2.4. The following diagrams are commutative:
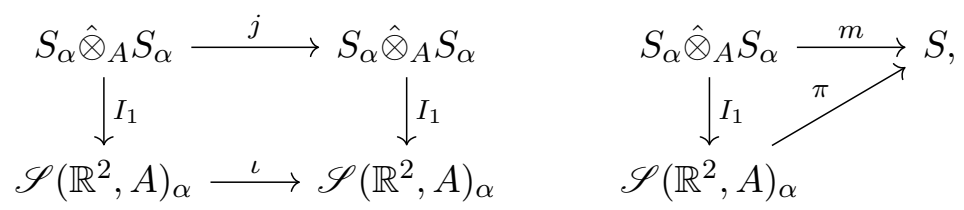

where

$$
\begin{aligned}
\iota(F)(x, y) & =\left(\frac{\partial F}{\partial x}-\frac{\partial F}{\partial y}\right)(x, y)+\alpha_{0}^{\prime}(F(x, y)) & & \text { for any } F \in \mathscr{S}\left(\mathbb{R}^{2}, A\right) \\
\pi(F)(x) & =\int_{\mathbb{R}} \alpha_{y}(F(y, x-y)) \mathrm{d} y & & \text { for any } F \in \mathscr{S}\left(\mathbb{R}^{2}, A\right)
\end{aligned}
$$


Proof. It is obvious that $\iota$ and $\rho$ are continuous, so we can assume that $F(x, y)=G(x) H(y)$ for some $G, H \in S:$

$$
\begin{aligned}
& I_{1} \circ j \circ I_{1}^{-1}(F)(x, y)=I_{1} \circ j(T G \otimes H)(x, y)=I_{1}\left((T G)^{\prime}(x) H(y)-(T G)(x)\left(T\left(T^{-1} H\right)(y)\right)\right)= \\
& =T^{-1}\left((T G)^{\prime}(x)\right) H(y)-G(x) T\left(\left(T^{-1} H\right)^{\prime}(y)\right) \stackrel{\underline{\underline{6}}}{=}\left(G^{\prime}(x)+\alpha_{0}^{\prime}(G(x))\right) H(y)-G(x)\left(H^{\prime}(y)-\alpha_{0}^{\prime}(H(y))\right)= \\
& =\left(\frac{\partial F}{\partial x}-\frac{\partial F}{\partial y}\right)(x, y)+\alpha_{0}^{\prime}(G(x)) H(y)+G(x) \alpha_{0}^{\prime}(H(y))=\left(\frac{\partial F}{\partial x}-\frac{\partial F}{\partial y}\right)(x, y)+\left.\frac{\mathrm{d}}{\mathrm{d} t} \alpha_{t}(G(x) H(y))\right|_{t=0} . \\
& \quad m \circ I_{1}^{-1}(F)(x)=\int_{R} T G(y) \alpha_{y}(H(x-y)) \mathrm{d} y=\int_{R} \alpha_{y}(G(y) H(x-y)) \mathrm{d} y=\pi(F)(x) .
\end{aligned}
$$

Now we can construct the right inverse maps for $\rho$.

Lemma 2.5. Fix a function $\varphi \in C_{c}^{\infty}(\mathbb{R})$ with $\int_{\mathbb{R}} \varphi(t) \mathrm{d} t=1$. Define the maps

$$
\begin{array}{ll}
\rho_{x}: S_{\alpha} \longrightarrow \mathscr{S}\left(\mathbb{R}^{2}, A\right)_{\alpha}, & \rho_{x}(F)(x, y)=\varphi(y) \alpha_{-x}(F(x+y)), \\
\rho_{y}: S_{\alpha} \longrightarrow \mathscr{S}\left(\mathbb{R}^{2}, A\right)_{\alpha}, & \rho_{y}(F)(x, y)=\varphi(x) \alpha_{-x}(F(x+y)) .
\end{array}
$$

Then $\rho_{x}$ is a $\mathscr{S}(\mathbb{R}, A ; \alpha)$ - $A$ - $\hat{\otimes}$-bimodule homomorphism, and $\rho_{y}$ is a $A$ - $\mathscr{S}(\mathbb{R}, A ; \alpha)$ - $\hat{\otimes}$-bimodule homomorphism. Moreover, we have

$$
\pi \circ \rho_{x}=\pi \circ \rho_{y}=\operatorname{Id}_{S_{\alpha}} .
$$

As a corollary, the algebra $\mathscr{S}(\mathbb{R}, A ; \alpha)$ is projective as a left and right $\mathscr{S}(\mathbb{R}, A ; \alpha)$ - $\hat{\otimes}$ module.

Proof. For any $F, H \in \mathscr{S}(\mathbb{R}, A ; \alpha), a \in A$ we have

$$
\begin{aligned}
\rho_{x}\left(H *_{\alpha} F\right)(x, y) & =\varphi(y) \int_{\mathbb{R}} \alpha_{-x}(H(z)) \alpha_{z-x}(F(x+y-z)) \mathrm{d} z \\
H \circ \rho_{x}(F)(x, y) & =\int_{\mathbb{R}} \alpha_{-x}(H(z)) \rho_{x}(F)(x-z, y) \mathrm{d} z=\int_{\mathbb{R}} \varphi(y) \alpha_{-x}(H(z)) \alpha_{z-x}(F(x+y-z)) \mathrm{d} z \\
\rho_{x}(F \circ a)(x, y) & =\varphi(y) \alpha_{-x}((F \circ a)(x+y))=\varphi(y) F(x+y) \alpha_{y}(a)=\left(\rho_{x}(F)\right) \circ a \\
\left(\pi \circ \rho_{x}\right)(F)(x) & =\int_{\mathbb{R}} \alpha_{y}\left(\rho_{x}(F)(y, x-y)\right) \mathrm{d} y=\int_{\mathbb{R}} \varphi(x-y) F(x) \mathrm{d} y=F(x) \int_{\mathbb{R}} \varphi(x-y) \mathrm{d} y=F(x) . \\
\rho_{y}\left(F *_{\alpha} H\right)(x, y) & =\varphi(x) \int_{\mathbb{R}} \alpha_{-x}(F(z)) \alpha_{z-x}(H(x+y-z)) \mathrm{d} z=\int_{\mathbb{R}} \varphi(x) \alpha_{-x}(F(z+x)) \alpha_{z}(H(y-z)) \mathrm{d} z \\
\rho_{y}(F) \circ H(x, y) & =\int_{\mathbb{R}} \rho_{y}(F)(x, z) \alpha_{z}(H(y-z)) \mathrm{d} z=\int_{\mathbb{R}} \varphi(x) \alpha_{-x}(F(x+z)) \alpha_{z}(H(y-z)) \mathrm{d} z \\
\rho_{y}(a \circ F)(x, y) & =\varphi(x) \alpha_{-x}((a \circ F)(x+y))=\varphi(x) \alpha_{-x}(a) F(x+y)=\left(a \circ \rho_{y}(F)\right)(x, y) \\
\left(\pi \circ \rho_{y}\right)(F)(x) & =\int_{\mathbb{R}} \alpha_{y}\left(\rho_{y}(F)(y, x-y)\right) \mathrm{d} y=\int_{\mathbb{R}} \varphi(y) F(x) \mathrm{d} y=F(x) .
\end{aligned}
$$

Lemma 2.6. Fix a function $\varphi \in C_{c}^{\infty}(\mathbb{R})$ with $\int_{\mathbb{R}} \varphi(t) \mathrm{d} t=1$. Define the maps

$$
\begin{aligned}
& \beta_{x}: \mathscr{S}\left(\mathbb{R}^{2}, A\right)_{\alpha} \longrightarrow \mathscr{S}\left(\mathbb{R}^{2}, A\right)_{\alpha}, \\
& \beta_{y}: \mathscr{S}\left(\mathbb{R}^{2}, A\right)_{\alpha} \longrightarrow \mathscr{S}\left(\mathbb{R}^{2}, A\right)_{\alpha}, \\
& \beta_{x}(F)(x, y)=\int_{-\infty}^{x}\left(\alpha_{t-x}(F(t, x+y-t))-\varphi(x+y-t) \int_{\mathbb{R}} \alpha_{z-x}(F(z, x+y-z)) \mathrm{d} z\right) \mathrm{d} t \\
& \beta_{y}(F)(x, y)=\int_{-\infty}^{x}\left(\alpha_{t-x}(F(t, x+y-t))-\varphi(t) \int_{\mathbb{R}} \alpha_{z-x}(F(z, x+y-z)) \mathrm{d} z\right) \mathrm{d} t
\end{aligned}
$$

Then $\beta_{x}$ is a $\mathscr{S}(\mathbb{R}, A ; \alpha)$-A- $\hat{\otimes}$-bimodule homomorphism, and $\beta_{y}$ is a $A$ - $\mathscr{S}(\mathbb{R}, A ; \alpha)$ - $\hat{\otimes}$-bimodule homomorphism. Moreover, we have

$$
\beta_{x} \circ \iota=\beta_{y} \circ \iota=\operatorname{Id}_{\mathscr{S}\left(\mathbb{R}^{2}, A\right)_{\alpha}} .
$$


Proof. We'll start by proving that $\beta_{x}$ is well-defined: it is not entirely obvious from the construction that these integrals define functions which belong to $\mathscr{S}\left(\mathbb{R}^{2}, A\right)$. Let us prove that the corresponding integral over $\mathbb{R}$ equals zero, then we can use the vector-valued version of the Haramard's lemma to prove that the antiderivative lies in $\mathscr{S}\left(\mathbb{R}^{2}, A\right)$, as well.

$$
\begin{aligned}
& \int_{\mathbb{R}}\left(\alpha_{t-x}(F(t, x+y-t))-\varphi(x+y-t) \int_{\mathbb{R}} \alpha_{z-x}(F(z, x+y-z)) \mathrm{d} z\right) \mathrm{d} t= \\
& =\int_{\mathbb{R}} \alpha_{t-x}(F(t, x+y-t)) \mathrm{d} t-\int_{\mathbb{R}}\left(\varphi(x+y-t) \int_{\mathbb{R}} \alpha_{z-x}(F(z, x+y-z)) \mathrm{d} z\right) \mathrm{d} t= \\
& =\int_{\mathbb{R}} \alpha_{t-x}(F(t, x+y-t)) \mathrm{d} t-\int_{\mathbb{R}} \varphi(x+y-t) \mathrm{d} t \int_{\mathbb{R}} \alpha_{z-x}(F(z, x+y-z)) \mathrm{d} z= \\
& =\int_{\mathbb{R}} \alpha_{t-x}(F(t, x+y-t)) \mathrm{d} t-\int_{\mathbb{R}} \alpha_{z-x}(F(z, x+y-z)) \mathrm{d} z=0
\end{aligned}
$$

Now we can prove that $\beta_{x}$ is a $\hat{\otimes}$-bimodule homomorphism. We notice that for every $H \in S, F \in$ $\mathscr{S}\left(\mathbb{R}^{2}, A\right)$ we have

$$
\begin{aligned}
\alpha_{t-x}((H \circ F)(t, x+y-t)) & =\alpha_{t-x}\left(\int_{\mathbb{R}} \alpha_{-t}(H(s)) F(t-s, x+y-t) \mathrm{d} s\right)= \\
& =\int_{\mathbb{R}} \alpha_{-x}(H(s)) \alpha_{t-x}(F(t-s, x+y-t)) \mathrm{d} s
\end{aligned}
$$

therefore, we have

$$
\begin{aligned}
& \beta_{x}(H \circ F)(x, y)=\int_{-\infty}^{x}\left(\alpha_{t-x}(H \circ F(t, x+y-t))-\varphi(x+y-t) \int_{\mathbb{R}} \alpha_{z-x}(H \circ F(z, x+y-z)) \mathrm{d} z\right) \mathrm{d} t= \\
& =\int_{-\infty}^{x}\left(\int_{\mathbb{R}} \alpha_{-x}(H(s)) \alpha_{t-x}(F(t-s, x+y-t)) \mathrm{d} s-\varphi(x+y-t) \int_{\mathbb{R}} \int_{\mathbb{R}} \alpha_{-x}(H(s)) \alpha_{z-x}(F(z-s, x+y-z)) \mathrm{d} s \mathrm{~d} z\right) \mathrm{d} t= \\
& =\int_{-\infty}^{x}\left(\int_{\mathbb{R}} \alpha_{-x}(H(s)) \alpha_{t-x}(F(t-s, x+y-t)) \mathrm{d} s-\varphi(x+y-t) \int_{\mathbb{R}} \alpha_{-x}(H(s)) \int_{\mathbb{R}} \alpha_{z-x}(F(z-s, x+y-z)) \mathrm{d} z \mathrm{~d} s\right) \mathrm{d} t= \\
& =\int_{-\infty}^{x}\left(\int_{\mathbb{R}} \alpha_{-x}(H(s))\left(\alpha_{t-x}(F(t-s, x+y-t))-\varphi(x+y-t) \int_{\mathbb{R}} \alpha_{z-x}(F(z-s, x+y-z)) \mathrm{d} z\right) \mathrm{d} s\right) \mathrm{d} t= \\
& =\int_{\mathbb{R}}\left(\int_{-\infty}^{x} \alpha_{-x}(H(s))\left(\alpha_{t-x}(F(t-s, x+y-t))-\varphi(x+y-t) \int_{\mathbb{R}} \alpha_{z-x}(F(z-s, x+y-z)) \mathrm{d} z\right) \mathrm{d} t\right) \mathrm{d} s= \\
& =\int_{\mathbb{R}} \alpha_{-x}(H(s))\left(\int_{-\infty}^{x}\left(\alpha_{t-x}(F(t-s, x+y-t))-\varphi(x+y-t) \int_{\mathbb{R}} \alpha_{z-x}(F(z-s, x+y-z)) \mathrm{d} z\right) \mathrm{d} t\right) \mathrm{d} s= \\
& =\int_{\mathbb{R}} \alpha_{-x}(H(s))\left(\int_{-\infty}^{x}\left(\alpha_{t+s-x}(F(t, x+y-t-s))-\varphi(x+y-t-s) \int_{\mathbb{R}} \alpha_{z+s-x}(F(z, x+y-z-s)) \mathrm{d} z\right) \mathrm{d} t\right) \mathrm{d} s= \\
& =\int_{\mathbb{R}} \alpha_{-x}(H(s)) \beta_{x}(F)(x-s, y) \mathrm{d} s=\left(H \circ \beta_{x}(F)\right)(x, y), \\
& \beta_{x}(F \circ a)(x, y)=\int_{-\infty}^{x}\left(\alpha_{t-x}((F \circ a)(t, x+y-t))-\varphi(x+y-t) \int_{\mathbb{R}} \alpha_{z-x}((F \circ a)(z, x+y-z)) \mathrm{d} z\right) \mathrm{d} t= \\
& =\int_{-\infty}^{x}\left(\alpha_{t-x}(F(t, x+y-t)) \alpha_{y}(a)-\varphi(x+y-t) \int_{\mathbb{R}} \alpha_{z-x}(F(z, x+y-z)) \alpha_{y}(a) \mathrm{d} z\right) \mathrm{d} t= \\
& =\left(\int_{-\infty}^{x}\left(\alpha_{t-x}(F(t, x+y-t))-\varphi(x+y-t) \int_{\mathbb{R}} \alpha_{z-x}(F(z, x+y-z)) \mathrm{d} z\right) \mathrm{d} t\right) \alpha_{y}(a)=\left(\beta_{x}(F) \circ a\right)(x, y) .
\end{aligned}
$$

To check that $\beta_{x}$ is the right inverse to $\iota$, we have to assume that $F(x, y)=G(x) H(y)$. First of all, notice that

$$
\begin{aligned}
& \frac{\mathrm{d}}{\mathrm{d} t}\left(\alpha_{t-x}(F(t, x+y-t))\right)=\frac{\mathrm{d}}{\mathrm{d} t}\left(\alpha_{t-x}(G(t)) \alpha_{t-x}(H(x+y-t))\right)= \\
& =\left(\alpha_{t-x}\left(G^{\prime}(t)\right)+\alpha_{t-x}^{\prime}(G(t))\right) \alpha_{t-x}(H(x+y-t))+\alpha_{t-x}(G(t))\left(\alpha_{t-x}^{\prime}(H(x+y-t))-\alpha_{t-x}\left(H^{\prime}(x+y-t)\right)\right)= \\
& =\alpha_{t-x}\left(G^{\prime}(t) H(x+y-t)+\alpha_{0}^{\prime}(G(t)) H(x+y-t)+G(t) \alpha_{0}^{\prime}(H(x+y-t))-G(t) H^{\prime}(x+y-t)\right) \stackrel{\underline{\underline{6}}}{\underline{\underline{6}}} \alpha_{t-x}\left(T^{-1}\left((T G)^{\prime}\right)(t) H(x+y-t)-G(t) T\left(\left(T^{-1} H\right)^{\prime}\right)(x+y-t)\right)=\alpha_{t-x}(\iota(F)(t, x+y-t)) .
\end{aligned}
$$


Therefore, we have

$$
\begin{aligned}
& \left(\beta_{x} \circ \iota(F)\right)(x, y)=\int_{-\infty}^{x}\left(\alpha_{t-x}(\iota(F)(t, x+y-t))-\varphi(x+y-t) \int_{\mathbb{R}} \alpha_{z-x}(\iota(F)(z, x+y-z)) \mathrm{d} z\right) \mathrm{d} t= \\
& =\int_{-\infty}^{x}\left(\frac{\mathrm{d}}{\mathrm{d} t}\left(\alpha_{t-x}(F(t, x+y-t))\right)-\varphi(x+y-t) \int_{\mathbb{R}} \frac{\mathrm{d}}{\mathrm{d} z}\left(\alpha_{z-x}(F(z, x+y-z))\right) \mathrm{d} z\right) \mathrm{d} t=F(x, y) .
\end{aligned}
$$

The necessary computations for $\beta_{y}$ are, essentially, the same.

By combining the Lemmas 2.1 - 2.6. we can formulate the following theorem:

Theorem 2.2. Let $A$ be a self-induced Fréchet-Arens-Michael algebra with a smooth $m$-tempered action $\alpha$ of $\mathbb{R}$ on $A$. Then the following diagram is commutative, moreover, the rows are short exact sequences of $\mathscr{S}(\mathbb{R}, A ; \alpha)$-bimodules which split in the categories $\mathscr{S}(\mathbb{R}, A ; \alpha)-A$-Mod $(\mathbf{F r})$ and $A-\mathscr{S}(\mathbb{R}, A ; \alpha)-\operatorname{Mod}(\mathbf{F r})$ :

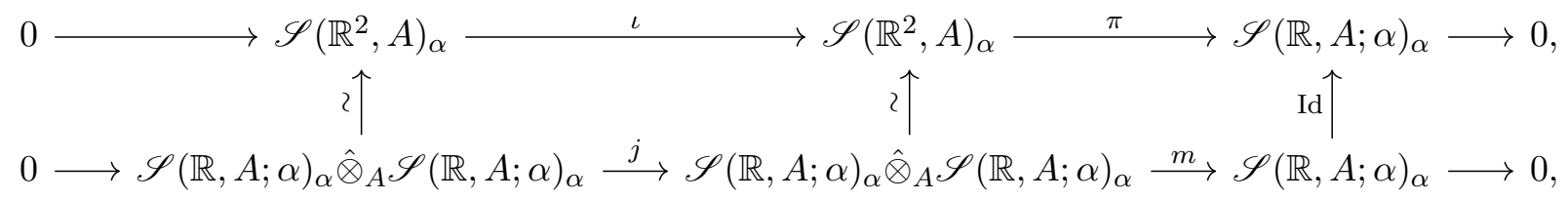

where

$$
\begin{aligned}
\iota(F)(x, y) & =\left(\frac{\partial F}{\partial x}-\frac{\partial F}{\partial y}\right)(x, y)+\alpha_{0}^{\prime}(F(x, y)) & \text { for any } F \in \mathscr{S}\left(\mathbb{R}^{2}, A\right) \\
\pi(F)(x) & =\int_{\mathbb{R}} \alpha_{y}(F(y, x-y)) \mathrm{d} y & \text { for any } F \in \mathscr{S}\left(\mathbb{R}^{2}, A\right) \\
j(F \otimes G) & =F^{\prime} \otimes G-F \otimes T\left(\left(T^{-1} G\right)^{\prime}\right) & \text { for any } F, G \in \mathscr{S}(\mathbb{R}, A ; \alpha) \\
m(F \otimes G) & =F *_{\alpha} G & \text { for any } F, G \in \mathscr{S}(\mathbb{R}, A ; \alpha) .
\end{aligned}
$$

Proof. In the previous lemmas we have constructed the sections $\rho_{x}, \rho_{y}, \beta_{x}, \beta_{y}$. The only thing that is left to check that $\iota \circ \beta_{x}+\rho_{x} \circ \pi=\iota \circ \beta_{y}+\rho_{y} \circ \pi=\operatorname{Id}_{\mathscr{S}\left(\mathbb{R}^{2}, A\right)_{\alpha}}$, then we use [Hel86, Proposition 3.1.8].

For any $F(x, y) \in \mathscr{S}\left(\mathbb{R}^{2}, A\right)_{\alpha}$ we have

$$
\begin{aligned}
& \left(\iota \circ \beta_{x}\right)(F)(x, y)=\iota\left(\int_{-\infty}^{x}\left(\alpha_{t-x}(F(t, x+y-t))-\varphi(x+y-t) \int_{\mathbb{R}} \alpha_{z-x}(F(z, x+y-z)) \mathrm{d} z\right) \mathrm{d} t\right)= \\
& =F(x, y)-\varphi(y) \int_{\mathbb{R}} \alpha_{z-x}(F(z, x+y-z)) \mathrm{d} z+ \\
& +\int_{-\infty}^{x}\left(\frac{\partial}{\partial x}-\frac{\partial}{\partial y}\right)\left(\alpha_{t-x}(F(t, x+y-t))-\varphi(x+y-t) \int_{\mathbb{R}} \alpha_{z-x}(F(z, x+y-z)) \mathrm{d} z\right) \mathrm{d} t+ \\
& +\int_{-\infty}^{x}\left(\alpha_{t-x}^{\prime}(F(t, x+y-t))-\varphi(x+y-t) \int_{\mathbb{R}} \alpha_{z-x}^{\prime}(F(z, x+y-z)) \mathrm{d} z\right) \mathrm{d} t= \\
& =F(x, y)-\varphi(y) \int_{\mathbb{R}} \alpha_{z-x}(F(z, x+y-z)) \mathrm{d} z+ \\
& +\int_{-\infty}^{x}\left(-\alpha_{t-x}^{\prime}(F(t, x+y-t))+\varphi(x+y-t) \int_{\mathbb{R}} \alpha_{z-x}^{\prime}(F(z, x+y-z)) \mathrm{d} z\right) \mathrm{d} t+ \\
& +\int_{-\infty}^{x}\left(\alpha_{t-x}^{\prime}(F(t, x+y-t))-\varphi(x+y-t) \int_{\mathbb{R}} \alpha_{z-x}^{\prime}(F(z, x+y-z)) \mathrm{d} z\right) \mathrm{d} t= \\
& =F(x, y)-\varphi(y) \int_{\mathbb{R}} \alpha_{z-x}(F(z, x+y-z)) \mathrm{d} z, \\
& \quad\left(\rho_{x} \circ \pi\right)(F)(x)=\varphi(y) \alpha_{-x}(\pi(F)(x+y))=\varphi(y) \int_{\mathbb{R}} \alpha_{z-x}(F(z, x+y-z)) \mathrm{d} z,
\end{aligned}
$$

therefore, we have

$$
\iota \circ \beta_{x}+\rho_{x} \circ \pi(F)(x, y)=F(x, y) .
$$

The argument for $\iota \circ \beta_{y}+\rho_{y} \circ \pi$ is similar. 
In the case $G=\mathbb{T}$ we obtain the following theorem.

Theorem 2.3. Let $A$ be a self-induced Fréchet-Arens-Michael algebra with a smooth $m$-tempered action $\alpha$ of $\mathbb{T}$ on $A$. Then the following diagram is commutative, moreover, the rows are short exact sequences of $C^{\infty}(\mathbb{T}, A ; \alpha)$-bimodules which split in the categories $C^{\infty}(\mathbb{T}, A ; \alpha)-A$-Mod(Fr) and $A-C^{\infty}(\mathbb{T}, A ; \alpha)-\operatorname{Mod}(\mathbf{F r}):$

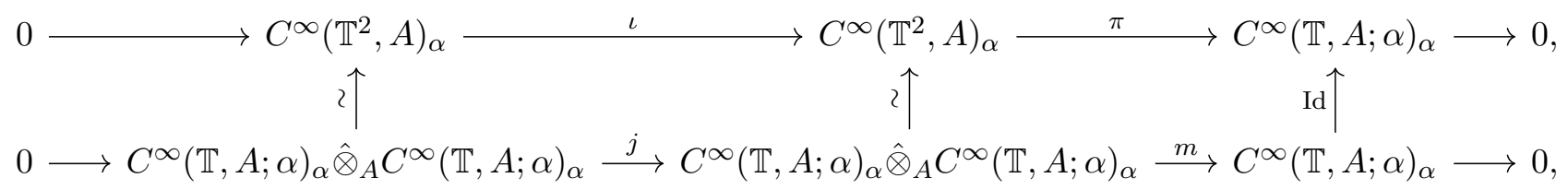

where

$$
\begin{array}{rlrl}
\iota(F)(x, y) & =\left(\frac{\partial F}{\partial x}-\frac{\partial F}{\partial y}\right)(x, y)+\alpha_{0}^{\prime}(F(x, y)) & & \text { for any } F \in C^{\infty}\left(\mathbb{T}^{2}, A\right) \\
\pi(F)(x) & =\int_{\mathbb{T}} \alpha_{y}(F(y, x-y)) \mathrm{d} y & & \text { for any } F \in C^{\infty}\left(\mathbb{T}^{2}, A\right) \\
j(F \otimes G) & =F^{\prime} \otimes G-F \otimes T\left(\left(T^{-1} G\right)^{\prime}\right) & \text { for any } F, G \in C^{\infty}(\mathbb{T}, A ; \alpha) \\
m(F \otimes G) & =F *_{\alpha} G & \text { for any } F, G \in C^{\infty}(\mathbb{T}, A ; \alpha) .
\end{array}
$$

\section{Obtaining upper estimates for homological dimensions of smooth crossed products by $\mathbb{R}$ and $\mathbb{T}$}

Remark. Again, we provide the proofs only for the case $G=\mathbb{R}$, but the same arguments work for $G=\mathbb{T}$, as well.

Here we adapt the arguments in [Kos17], which were used to obtain the upper estimates to the non-unital case.

Definition 3.1. Let $A$ be a $\hat{\otimes}$-algebra. Then a $\hat{\otimes}$-algebra $S$ together with a $A$ - $\hat{\otimes}$-bimodule structure is called an $A$ - $\hat{\otimes}$-algebra if $S \in A$-mod- $S$ and $S \in S$-mod- $A$.

This definition works as expected in the unital case.

Proposition 3.1. Let $A$ be a unital $\hat{\otimes}$-algebra. A $A$ - $\hat{\otimes}$-bimodule structure on a unital $A$ - $\hat{\otimes}$-algebra $S$ is uniquely defined by a (unital) algebra homomorphism $\eta: A \rightarrow S$ :

$$
a \circ s=\eta(a) s, \quad s \circ a=s \eta(a)
$$

for every $a \in A, s \in S$.

Proof. Define $\eta$ as follows: $\eta(a)=a \circ 1_{S}=1_{S} \circ a$. It is easy to see that $\eta$ is an algebra homomorphism. Also, we have

$$
\begin{gathered}
\eta(a) s=\left(a \circ 1_{S}\right) s=a \circ\left(1_{S} s\right)=a \circ s, \\
s \eta(a)=s\left(a \circ 1_{S}\right)=s\left(1_{S} \circ a\right)=\left(s 1_{S}\right) \circ a=s \circ a
\end{gathered}
$$

for any $a \in A, s \in S$.

As a corollary from Lemma 2.1 we get that the $A$ - $\hat{\otimes}$-bimodule structure on $S_{\alpha}$ makes $\mathscr{S}(\mathbb{R}, A ; \alpha)$ into a $A$-बे-algebra.

Proposition 3.2. Let $A$ be a Fréchet-Arens-Michael algebra, and let $\alpha$ be a $m$-tempered action of $\mathbb{R}$ on $A$. Consider the following multiplication on $\mathscr{S}(\mathbb{R}, A)$ :

$$
\left(f *_{\alpha}^{\prime} g\right)(x)=\int_{\mathbb{R}} \alpha_{-y}(f(x-y)) g(y) \mathrm{d} y .
$$

Then the following locally convex algebra isomorphism takes place:

$$
i: \mathscr{S}(\mathbb{R}, A ; \alpha) \rightarrow\left(\mathscr{S}(\mathbb{R}, A), *_{\alpha}^{\prime}\right), \quad i(f)(x)=\alpha_{-x}(f(x)) .
$$


Proof. The mapping $i$ is, obviously, a topological isomorphism of locally convex spaces. Now notice that

$$
\begin{aligned}
\left(i(f) *^{\prime} i(g)\right)(x) & =\int_{\mathbb{R}} \alpha_{-y}(i(f)(x-y)) i(g)(y) \mathrm{d} y=\int_{\mathbb{R}} \alpha_{-x}(f(x-y)) \alpha_{-y}(g(y)) \mathrm{d} y= \\
& =\int_{\mathbb{R}} \alpha_{-x}(f(-y)) \alpha_{-x-y}(g(y+x)) \mathrm{d} y=\alpha_{-x}\left(\int_{\mathbb{R}} f(-y) \alpha_{-y}(g(y+x)) \mathrm{d} y\right)= \\
& =i(f * g)(x),
\end{aligned}
$$

therefore, $i$ is an algebra homomorphism.

Corollary 3.1. Define the $A$ - $\hat{\otimes}$-bimodule and $\left(\mathscr{S}(\mathbb{R}, A), *_{\alpha}^{\prime}\right)$ - $\hat{\otimes}$-bimodule ${ }_{\alpha^{-1}} \mathscr{S}(\mathbb{R}, A ; \alpha)={ }_{\alpha^{-1}} S$ as follows: $\alpha^{-1} S$ coincides with $\mathscr{S}(\mathbb{R}, A)$ as a LCS, and

$$
\begin{aligned}
& (F \circ a)(x)=F(x) a, \quad a \circ F(x)=\alpha_{-x}(a) F(x) \quad \text { for any } a \in A, F \in \in_{\alpha^{-1}} S \\
& (F \circ G)(x)=\left(F *_{\alpha}^{\prime} G\right)(x), \quad(G \circ F)(x)=\left(G *_{\alpha}^{\prime} F\right)(x) \quad \text { for any } F \in \alpha_{\alpha^{-1}} S, G \in\left(\mathscr{S}(\mathbb{R}, A), *_{\alpha}^{\prime}\right) \text {. }
\end{aligned}
$$

Then the map

$$
S_{\alpha} \longrightarrow \alpha^{-1} S, \quad F(x) \longmapsto \alpha_{-x}(F(x)),
$$

is an isomorphism of $A$ - $\hat{\otimes}$-bimodules. As a corollary, $S_{\alpha}$ is projective as a left and right $A$ - $\hat{\otimes}$-bimodule.

Definition 3.2. Let $A$ be a $\hat{\otimes}$-algebra. A left $A$ - $\hat{\otimes}$-module $M$ is called essential, if the canonical morphism $\eta_{M}: A \hat{\otimes}_{A} M \longrightarrow M$ is an isomorphism of left $A$ - $\hat{\otimes}$-modules.

Lemma 3.1. Let $A$ be a self-induced Fréchet-Arens-Michael algebra together with an $m$-tempered $\mathbb{R}$-action $\alpha$ of $\mathbb{R}$. Then the module $\mathscr{S}(\mathbb{R}, A ; \alpha)_{\alpha}$ is an essential left and right $A$ - $\hat{\otimes}$-module.

Example 3.1. If $A$ is a self-induced $\hat{\otimes}$-algebra, then for any left $A$ - $\hat{\otimes}$-module $M$ the module $A \hat{\otimes}_{A} M$ is essential.

Proof. Recall that the mapping $i: A \hat{\otimes} \mathscr{S}(\mathbb{R})_{\alpha} \longrightarrow \mathscr{S}(\mathbb{R}, A ; \alpha), i(a \otimes f)(x)=f(x) a$, is an isomorphism of left $A$ - $\hat{\otimes}$-modules. Therefore, we can write the following composition of isomorphisms:

$$
A \hat{\otimes}_{A} \mathscr{S}(\mathbb{R}, A ; \alpha)_{\alpha} \stackrel{i^{-1}}{\longrightarrow} A \hat{\otimes}_{A} A \hat{\otimes} \mathscr{S}(\mathbb{R}) \stackrel{m}{\rightarrow} A \hat{\otimes} \mathscr{S}(\mathbb{R}) \stackrel{i}{\rightarrow} \mathscr{S}(\mathbb{R}, A ; \alpha)_{\alpha}
$$

Notice that $i^{-1} \circ m \circ i(a \otimes f(x) b)=f(x) a b=\eta_{M}(a \otimes f(x) b)$, so $\eta_{M}$ coincides with $i^{-1} \circ m \circ i$ on a dense subset, therefore, $\eta_{M}$ is an isomorphism of left $A$ - $\hat{\otimes}$-modules. The same argument shows that ${ }_{\alpha^{-1}} \mathscr{S}(\mathbb{R}, A ; \alpha)$ is an essential right $A$ - $\hat{\otimes}$-module, but ${ }_{\alpha^{-1}} \mathscr{S}(\mathbb{R}, A ; \alpha) \cong \mathscr{S}(\mathbb{R}, A ; \alpha)_{\alpha}$.

Lemma 3.2. Let $A$ be a $\hat{\otimes}$-algebra and let $S$ be an $A$ - $\hat{\otimes}$-algebra.

(1) Let $X$ be a projective right $A$ - $\hat{\otimes}$-module. Then the module $X \hat{\otimes}_{A} S$ is a projective right $S$ $\hat{\otimes}$-module. Similarly, if $X$ is a projective left $A$ - $\hat{\otimes}$-module, then $S \hat{\otimes}_{A} X$ is a projective left $S$-बे-module.

(2) Let $X$ be a projective $A$ - $\hat{\otimes}$-bimodule. Then the module $S \hat{\otimes}_{A} X \hat{\otimes}_{A} S$ is a projective $S$ - $\hat{\otimes}$-bimodule.

Proof. (1) The module $X$ is projective, therefore, there is a retraction $\sigma: E \hat{\otimes} A_{+} \longrightarrow X$. But then the map

$$
E \hat{\otimes} S_{+} \longrightarrow E \hat{\otimes} S \cong E \otimes A_{+} \hat{\otimes}_{A} S \longrightarrow X \hat{\otimes}_{A} S
$$

is a composition of retractions, and retracts of free modules are projective. Proof for the left modules is similar.

(2) The bimodule $X$ is projective, therefore, there is a retraction $\sigma: A_{+} \hat{\otimes} E \hat{\otimes} A_{+} \longrightarrow X$. Then the map

$$
S \hat{\otimes} E \hat{\otimes} S \longrightarrow S \hat{\otimes}_{A} A_{+} \hat{\otimes} E \hat{\otimes} A_{+} \hat{\otimes}_{A} S \longrightarrow S \hat{\otimes}_{A} X \hat{\otimes}_{A} S
$$

is a composition of retractions. Due to Hel86, Proposition 4.1.4], the $S$ - $\hat{\otimes}$-bimodule $S \hat{\otimes} E \hat{\otimes} S$ is projective, and retracts of projective modules are projective. 
Lemma 3.3. Let $A$ be a $\hat{\otimes}$-algebra. Also let $\{M, d\}$ denote an admissible sequence of right $A$ - $\hat{\otimes}$ modules. If $X$ is a projective left $A$ - $\hat{\otimes}$-module, then the complex $\left\{M \hat{\otimes}_{A} X, d \otimes \operatorname{Id}\right\}$ splits in LCS.

Similarly, for every projective right $A$ - $\hat{\otimes}$-module $X$ the complex $\{X \hat{\otimes} M, d \otimes \operatorname{Id}\}$ splits in LCS.

Proof. If $X$ were a free left $A$ - $\hat{\otimes}$-module, then the statement of the lemma would follow from the canonical isomorphism $M \hat{\otimes}_{A} A_{+} \hat{\otimes} E \cong M \hat{\otimes} X$ for some $E \in \mathbf{L C S}$. However, a retract of an admissible sequence is admissible, as well.

Lemma 3.4. Let $A$ be a $\hat{\otimes}$-algebra and let $S$ be an $A$ - $\hat{\otimes}$-algebra, which is projective as a left $A$ - $\hat{\otimes}$ module. Then we have

$$
\operatorname{dg}_{S^{o p}}\left(M \hat{\otimes}_{A} S\right) \leq \operatorname{dg}_{A^{o p}}(M) .
$$

If $S$ is projective as a right $A$ - $\hat{\otimes}$-module, then

$$
\operatorname{dg}_{S}\left(S \hat{\otimes}_{A} M\right) \leq \operatorname{dg}_{A}(M) .
$$

Proof. Suppose we have a projective resolution of $M$ in $\bmod -A$ :

$$
0 \longleftarrow M \stackrel{d_{0}}{\longleftarrow} P_{0} \longleftarrow d_{1} \cdots \longleftarrow P_{n} \longleftarrow 0 \longleftarrow \ldots
$$

Then due to Lemma 3.2 and 3.3 the following sequence is the projective resolution for $M \hat{\otimes}_{A} S$ in $\bmod -S$ :

$$
0 \longleftarrow M \hat{\otimes}_{A} S \stackrel{d_{0} \otimes \mathrm{Id}}{\longleftarrow} P_{0} \hat{\otimes}_{A} S \stackrel{d_{2} \otimes \mathrm{Id}}{\longleftarrow} \cdots \longleftarrow P_{n} \hat{\otimes}_{A} S \longleftarrow 0 \longleftarrow \ldots
$$

Therefore, $\operatorname{dg}_{S^{\text {op }}}\left(M \hat{\otimes}_{A} S\right) \leq \operatorname{dg}_{A^{\text {op }}}(M)$.

Lemma 3.5. Let $A$ be a self-induced Fréchet-Arens-Michael algebra together with a smooth $m$ tempered $\mathbb{R}$-action $\alpha$. Set $S=\mathscr{S}(\mathbb{R}, A ; \alpha)$. For any right $S$ - $\hat{\otimes}$-module $M$ we have the following estimate:

$$
\operatorname{dh}_{S^{\mathrm{op}}}\left(M \hat{\otimes}_{S} S_{\alpha}\right) \leq \operatorname{dh}_{A^{\mathrm{op}}}\left(M \hat{\otimes}_{S} S_{\alpha}\right)+1 \leq \mathrm{dg}\left(A^{\mathrm{op}}\right)+1 .
$$

And for any left $S$ - $\hat{\otimes}$-module $M$ we have

$$
\operatorname{dh}_{S}\left(S_{\alpha} \hat{\otimes} M\right) \leq \operatorname{dh}_{A}\left(S_{\alpha} \hat{\otimes} M\right)+1 \leq \operatorname{dg}(A)+1 .
$$

Proof. Due to the Theorem 2.2 we have the following sequence:

$$
0 \longrightarrow S_{\alpha} \hat{\otimes}_{A} S_{\alpha} \longrightarrow S_{\alpha} \hat{\otimes}_{A} S_{\alpha} \longrightarrow S_{\alpha} \longrightarrow 0 .
$$

By applying the functor $M \hat{\otimes}_{S}(-)$ to 12 , we get

$$
0 \longrightarrow M \hat{\otimes}_{S} S_{\alpha} \hat{\otimes}_{A} S_{\alpha} \longrightarrow M \hat{\otimes}_{S} S_{\alpha} \hat{\otimes}_{A} S_{\alpha} \longrightarrow M \hat{\otimes}_{S} S_{\alpha} \longrightarrow 0 .
$$

Obviously, this sequence is admissible, therefore, we can apply Lemma 3.4, because $S_{\alpha}$ is a projective $A-\hat{\otimes}$-module (Corollary 3.1).

$$
\operatorname{dh}_{S^{\mathrm{op}}}\left(M \hat{\otimes}_{S} S_{\alpha}\right) \leq \operatorname{dh}_{S^{\mathrm{op}}}\left(M \hat{\otimes}_{S} S_{\alpha} \hat{\otimes}_{A} S_{\alpha}\right)+1 \stackrel{[3.4}{\leq} \mathrm{dh}_{A^{\mathrm{op}}}\left(M \hat{\otimes}_{S} S_{\alpha}\right)+1 \leq \operatorname{dg}\left(A^{\mathrm{op}}\right)+1 .
$$

So, we have just obtained the upper bound for projective dimension of essential modules. To obtain an estimate for an arbitrary right $S$ - $\hat{\otimes}$-module, we use the method, described in the Lemmas 1-3 of the paper [OK84].

Theorem 3.1. Hel86, Theorem 5.2.1] Let $A$ be a $\hat{\otimes}$-algebra and let $X$ be a left $A$ - $\hat{\otimes}$ module. Then the following complex is admissible:

$$
0 \longleftarrow X \longleftarrow\left(A_{+} \hat{\otimes} X\right) \oplus\left(A \hat{\otimes}_{A} X\right) \stackrel{\delta_{0}}{\longleftarrow}\left(A_{+} \hat{\otimes}\left(A \hat{\otimes}_{A} X\right)\right) \oplus A \hat{\otimes} X \stackrel{\delta_{1}}{\longleftarrow} A \hat{\otimes}\left(A \hat{\otimes}_{A} X\right) \longleftarrow 0 .
$$

Moreover, this sequence is isomorphic to the tensor product of the following short admissible complexes:

$$
\begin{gathered}
0 \longleftarrow \operatorname{Im} \delta_{0} \stackrel{\delta_{0}}{\longleftarrow}\left(A_{+} \hat{\otimes}\left(A \hat{\otimes}_{A} X\right)\right) \oplus A \hat{\otimes} X \stackrel{\delta_{1}}{\longleftarrow} A \hat{\otimes}\left(A \hat{\otimes}_{A} X\right) \longleftarrow 0, \\
0 \longleftarrow X \longleftarrow\left(A_{+} \hat{\otimes} X\right) \oplus\left(A \hat{\otimes}_{A} X\right) \hookleftarrow \operatorname{Im} \delta_{0} \longleftarrow 0 .
\end{gathered}
$$


Notice that the modules $\left(A_{+} \hat{\otimes}\left(A \hat{\otimes}_{A} X\right)\right) \oplus A \hat{\otimes} X$ and $A \hat{\otimes}\left(A \hat{\otimes}_{A} X\right)$ are projective left $A$-modules, therefore, $\operatorname{dh}_{A}\left(\operatorname{Im} \delta_{0}\right) \leq 1$.

But then we also have

$$
\operatorname{dh}_{A}(X) \leq \max \left\{\operatorname{dh}_{A}\left(\left(A_{+} \hat{\otimes} X\right) \oplus\left(A \hat{\otimes}_{A} X\right)\right), \operatorname{dh}_{A}\left(\operatorname{Im} \delta_{0}\right)+1\right\} \leq \max \left\{\operatorname{dh}_{A}\left(A \hat{\otimes}_{A} X\right), 2\right\},
$$

for any $\hat{\otimes}$-algebra $A$ and a left $A$ - $\hat{\otimes}$-module $X$. Combining (15) with the Lemma 3.5, we get the following: for every left $S$ - $\hat{\otimes}$-module $M$ we have

$$
\operatorname{dh}_{S}(M) \stackrel{\text { (15) }}{\leq} \max \left\{\operatorname{dh}_{S}\left(S_{\alpha} \hat{\otimes}_{S} M\right), 2\right\} \stackrel{[3.5}{\leq} \max \{\operatorname{dgl}(A)+1,2\}=\max \{\operatorname{dgl}(A), 1\}+1 .
$$

Theorem 3.2. Let $A$ be a self-induced Fréchet-Arens-Michael algebra equipped with a smooth $m$ tempered $\mathbb{R}$-action $\alpha$. Then the following estimate takes place:

$$
\operatorname{dgl}(\mathscr{S}(\mathbb{R}, A ; \alpha)) \leq \max \{\operatorname{dgl}(A), 1\}+1 .
$$

The same result holds for $G=\mathbb{T}$ :

Theorem 3.3. Let $A$ be a self-induced Fréchet-Arens-Michael algebra equipped with a smooth $m$ tempered $\mathbb{T}$-action $\alpha$. Then the following estimate takes place:

$$
\operatorname{dgl}\left(C^{\infty}(\mathbb{T}, A ; \alpha)\right) \leq \max \{\operatorname{dgl}(A), 1\}+1 .
$$

\section{References}

[CQ95] J. Cuntz and D. Quillen. "Algebra Extensions and Nonsingularity". In: Journal of the American Mathematical Society 8.2 (1995).

[Fol99] Gerald B. Folland. Real analysis: modern techniques and their applications. 2nd ed. PAM. Wiley, 1999. ISBN: 0471317160,9780471317166.

[GG11] O. Gabriel and M. Grensing. "Six-Term Exact Sequences for Smooth Generalized Crossed Products". In: ArXiv e-prints (Nov. 2011). arXiv: 1111.2154 [math.KT],

[Hel86] A. Ya. Helemskii. The homology of Banach and topological algebras. Mathematics and Its Applications. Kluwer Academic Publishers, 1986. ISBN: 978-94-010-7560-2.

[Kos17] P. Kosenko. "Homological dimensions of analytic Ore extensions". In: ArXiv e-prints (Dec. 2017). arXiv: 1712.06177 [math.FA].

[Mal86] A. Mallios. Topological Algebras Selected Topics. North-Holland Mathematics Studies 124. Elsevier Science Ltd, 1986.

[Mey04] R. Meyer. "Embeddings of derived categories of bornological modules". In: ArXiv Mathematics e-prints (Oct. 2004). eprint: math/0410596.

[Nes14] Sergey Neshveyev. "Smooth crossed products of Rieffel's deformations". In: Letters in Mathematical Physics 104.3 (2014), pp. 361-371.

[OK84] OS Ogneva and A Ya Khelemskii. "Homological dimensions of certain algebras of principal (test) functions". In: Mathematical notes of the Academy of Sciences of the USSR 35.2 (1984), pp. 93-99.

[Pir08] A. Yu. Pirkovskii. "Arens-Michael envelopes, homological epimorphisms, and relatively quasi-free algebras". In: Transactions of the Moscow Mathematical Society 69 (2008).

[Pir12] A. Yu. Pirkovskii. "Homological dimensions and Van den Bergh isomorphisms for nuclear Fréchet algebras". In: Izvestiya: Mathematics 76.4 (2012).

[PS94] N. Christopher Phillips and Larry B. Schweitzer. "Representable K-theory of smooth crossed products by R and Z". In: Transactions of the American Mathematical Society 344.1 (1994), pp. 173-201. 
[Sch93] Larry B. Schweitzer. "Dense m-convex Fréchet subalgebras of operator algebra crossed products by Lie groups". In: International Journal of Mathematics 4.04 (1993), pp. 601-673.

P. Kosenko, Department of Mathematics, Higher School of Economics, Moscow, Usacheva str. 6 E-mail address, P. Kosenko: prkosenko@edu.hse.ru

Department of Mathematics, University of Toronto, 40 St. George St., Toronto, On, Canada

E-mail address: petr.kosenko@mail.utoronto.ca 